\title{
A mineral-scale investigation of the origin of the 2.6 Ma Füzes-tó basalt, Bakony-Balaton Highland Volcanic Field (Pannonian Basin, Hungary)
}

\author{
Éva Jankovics, Szabolcs Harangi \\ Department of Petrology and Geochemistry, \\ Eötvös Loránd University, Budapest
}

\author{
Theodoros Ntaflos \\ Department für Lithosphärenforschung \\ Universität Wien, Wien
}

\begin{abstract}
The alkaline basalt of the Füzes-tó scoria cone is the youngest volcanic product of the BakonyBalaton Highland Volcanic Field. The bombs and massive lava fragments are rich in various crystals, such as mantle-derived xenocrysts (olivine, orthopyroxene, clinopyroxene, spinel), high-pressure mineral phases (clinopyroxene) and phenocrysts (olivine, clinopyroxene). Peridotite xenoliths are also common. Ratios of incompatible trace elements $(\mathrm{Zr} / \mathrm{Nb}$ and $\mathrm{Nb} / \mathrm{Y})$ suggest that the primary magma was formed in the transitional spinel-garnet stability field, at the uppermost part of the asthenosphere. Magmatic spinel inclusions with low-Cr\# (22-35) in olivine phenocrysts can reflect a fertile peridotite source. The olivine, orthopyroxene, colourless clinopyroxene and spinel xenocrysts are derived from different depths of the subcontinental lithospheric mantle and their compositions resemble the mineral phases of the ultramafic xenoliths found in this region. The rarer green clinopyroxene cores of clinopyroxene phenocrysts could represent high-pressure products of crystallization from a more evolved melt than the host magma, or they could be derived from mafic lower crustal rocks. Crystallization of the basaltic magma resulted in olivine and clinopyroxene phenocrysts. Their compositions reflect polybaric crystallization with a final, strongly oxidized stage. The Füzes-tó basalt does not represent a certain magma composition, but a mixture of mineral phases having various origin and mantle-derived basaltic melt.
\end{abstract}

Key words: alkaline basalt, scoria cone, xenocryst, peridotite, lithospheric mantle

\section{Introduction}

Basalts are the most common volcanic rocks on the Earth. Each year, about 4 $\mathrm{km}^{3}$ of basaltic magma can reach the surface. They are formed by partial melting

\author{
Addresses: É. Jankovics, Sz. Harangi: H-1117 Budapest, Pázmány P. sétány 1C, Hungary \\ e-mail: jeva182@gmail.com \\ T. Ntaflos: Althanstrasse 14, 1090 Wien, Austria \\ Received: February 2, 2010; accepted: April 12, 2010
}


of the upper mantle and most of them arrive at the surface relatively rapidly (ascent rates are in the range of 0.1 to a few $\mathrm{m} / \mathrm{s}$; Spera 1984) and without significant differentiation. Thus, they provide important, unique information about the deep processes occurring in the Earth's interior and also about the nature of the upper mantle (Hofmann 1997). Evolution of basaltic magmas is usually simple due to their low volume, limited thermal budget and the rapid ascent. Compositions of the primary magmas could, however, have been modified either by incorporating rock and mineral fragments (xenoliths and xenocrysts) from the mantle and the crust and by deep-seated crystallization. The latter is not always obvious, since the early-stage crystallization products (e.g. clinopyroxene) are often absent from the erupted magma (Smith et al. 2008). Occasionally, various clinopyroxene assemblages in single basaltic rocks could reflect the complex history of basaltic magmas (Woodland and Jugo 2007).

In the Pannonian Basin (Fig. 1a), alkaline basalt volcanism took place from 11 Ma to $0.13 \mathrm{Ma}$, forming monogenetic volcanic fields, mostly at the peripheral areas. Compositions of the mafic rocks are regarded to be close to the primary melts; thus they are used to characterize the sublithospheric mantle beneath this region (Embey-Isztin et al. 1993, 2001a; Embey-Isztin and Dobosi 1995; Dobosi et

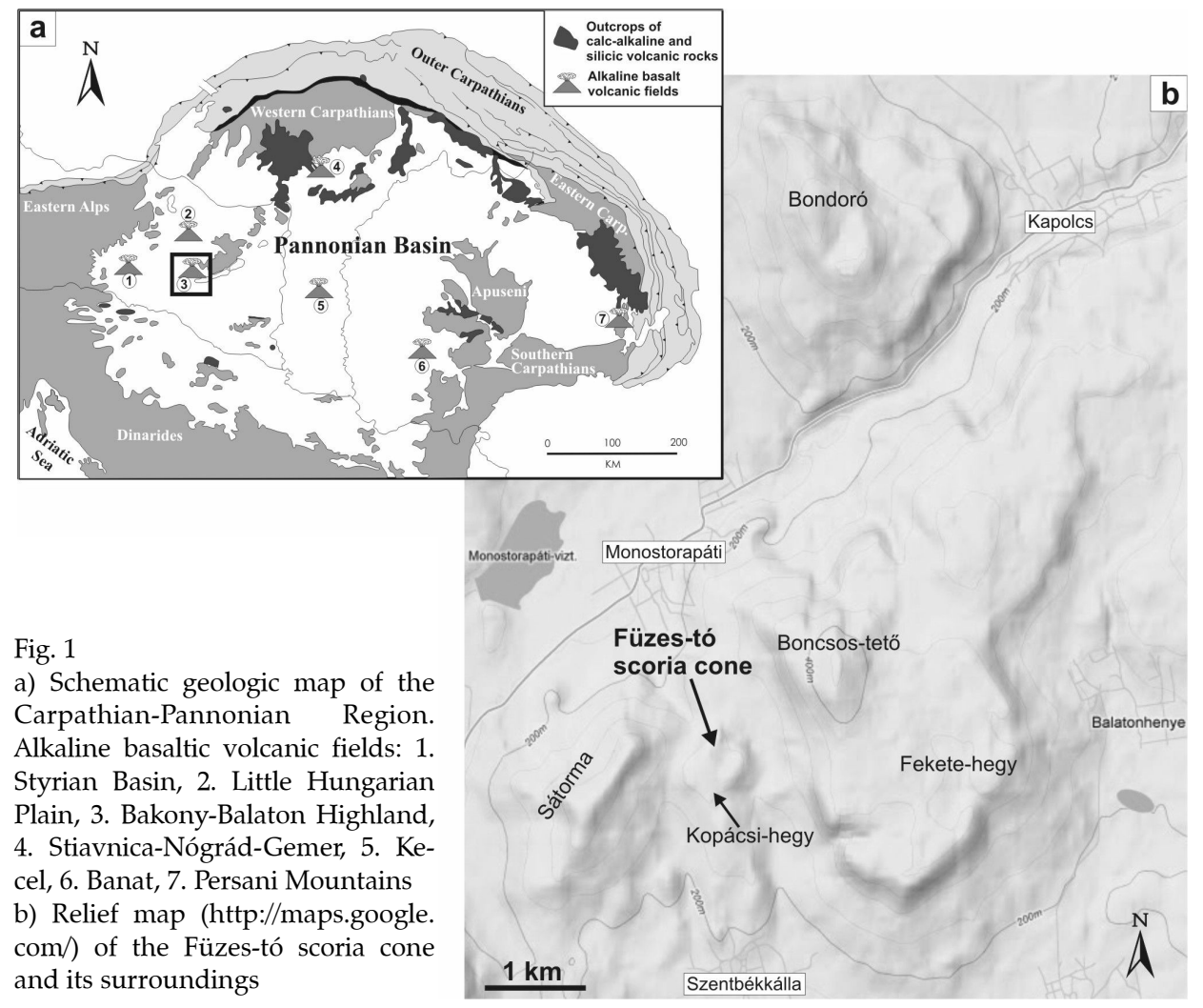


al. 1995; Harangi et al. 1994, 1995; Harangi 2001). Most of the basalts are olivinephyric, although clinopyroxene phenocrysts also occur in some localities. The complex zoning of the clinopyroxene (Dobosi 1989; Dobosi et al. 1991; Dobosi and Fodor 1992) implies the importance of this mineral in the early-stage crystallization in some of the mafic magmas. In this paper we describe a $2.6 \mathrm{Ma}$ basaltic occurrence from the Bakony-Balaton Highland Volcanic Field, western Pannonian Basin, and show that the mineral-scale investigation provides an insight into the deep processes occurring even in rapidly ascending basaltic magmas. The results pointr out that the bulk rock compositions of basalts should often be used with caution, since their mineral phases, being of different origins, can significantly modify it.

\section{Geologic background}

The Füzes-tó scoria cone is located in the middle of the Bakony-Balaton Highland Volcanic Field (BBHVF; Fig. 1a). It was formed during the last active period of this monogenetic volcanic field dated at 2.61 Ma (Wijbrans et al. 2007). This is the youngest age for the basalts of the BBHVF. This volcanic field comprises the largest number of volcanic centers and shows the greatest magma productivity among the Neogene-Quaternary alkaline basaltic volcanic fields of the Pannonian Basin (Embey-Isztin et al. 1993). The K/Ar (Balogh et al. 1982, 1986; Borsy et al. 1986; Balogh and Pécskay 2001) and ${ }^{40} \mathrm{Ar} /{ }^{39} \mathrm{Ar}$ dating (Wijbrans et al. 2007) implies that the duration of the volcanic activity was approximately 6 million years, from about $8 \mathrm{Ma}$ up to $2 \mathrm{Ma}$. According to the new high resolution ${ }^{40} \mathrm{Ar} /{ }^{39} \mathrm{Ar}$ data (Wijbrans et al. 2007), four main active periods are recognized, at about 7.95 Ma, 4.1 Ma, 3.8 Ma and 3.0 Ma.

The BBHVF has more than 50 eruptive centers; it consists of erosional remnants of different volcanic forms like maars, tuff rings, scoria cones, lava lakes and lava flows (shield volcanoes) (Jugovics 1968; Németh and Martin 1999; Martin et al. 2003). Most of the volcanic edifices are strongly eroded; often only their root zones and feeding channels have been preserved. The wide range of volcanic forms reflects the various mechanisms of volcanic eruptions. Here, almost all types of eruption of basaltic magmas have been recognized, from maar and tuff ring-forming phreatomagmatic activity through Strombolian and Hawaiian style eruptions to effusive events (Németh and Martin 1999; Németh et al. 2001).

The tectonic background of the alkaline basaltic magmatism is still a subject of debate. Hoernle et al. (1995) and Seghedi et al. (2004) suggested that localized mantle plume fingers beneath the Pannonian Basin could be responsible for the basaltic magmatism, whereas Harangi and Lenkey (2007) argued against the plume-related magmatism and suggested that asthenospheric mantle flow from below the thick Alpine regime could lead to partial melting of the strongly heterogeneous upper mantle. 


\section{Volcanological features}

The Füzes-tó scoria cone belongs to a complex volcanic area comprising the Sátorma, Fekete-hegy - Boncsos-tetô nested maar volcanic structures and overlying basaltic lava caps (Auer et al. 2007) and the Kopácsi-hegy - Ereszvény scoria cone and maar volcanoes (Fig. 1b). It is probably the least eroded primary volcanic form in the entire Balaton Highland (Csillag 2004). It was built on deposits of small-volume basaltic pyroclastic flows, base surges and fall-out beds (Németh and Szabó 1998). The volcanic edifice can still be recognized, although it is covered by vegetation (Fig. 2a). It is characterized by a well-preserved central depression, where occasionally a small pond is located. The inner slope around the central depression is covered by reddish basalt fragments with variable vesicularity. Plastic deformation structure can often be recognized in the basalts as alignments of vesicles and phenocrysts.

Well-preserved volcanic bombs of variable shape and size are common. There are numerous spindle bombs, rotational bombs (Fig. 2b), scoriaceous bombs (Fig. $2 \mathrm{c})$, agglutinated pyroclastics and breadcrust bombs. The size of the largest bomb is approximately $1 \mathrm{~m}$ (Fig. $2 \mathrm{~d}$ ). In the core of the bombs there are frequently dense ultramafic upper-mantle xenoliths (Fig. 2e), which are sometimes variably disaggregated. Furthermore, rounded peridotite nodules also occur; however, they are usually strongly altered. Some massive grey lava fragments can be also found in the area, which contain mm-sized xenocrysts and smaller fragments of disaggregated xenoliths. The abundance of scoriaceous and spatter rocks with ballistic bombs suggests explosive volcanic eruptions, such as Hawaiian and Strombolian lava fountaining.

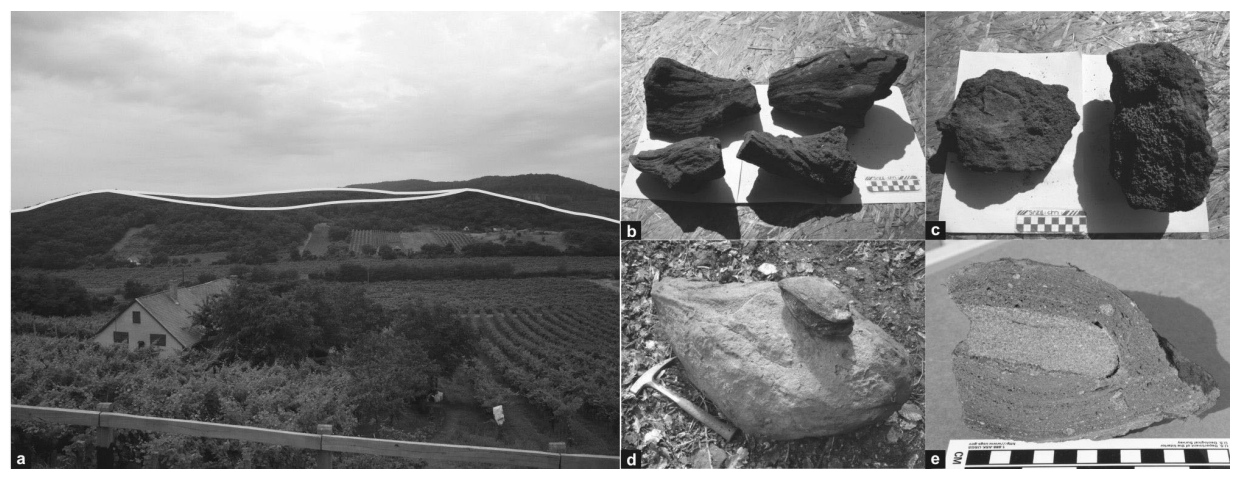

Fig. 2

a) The Füzes-tó scoria cone, whose well-preserved form is emphasized with a thick white line. The picture was made from the Boncsos-tető which lies to its east; b) Rotational bombs; c) Scoriaceous bombs which contain small xenoliths; d) The greatest bomb found in the Füzes-tó area, and there is a smaller spindle bomb on it; e) A split spindle bomb which encloses an altered xenolith; there are also several fragments and minerals of peridotites around it 


\section{Analytical methods}

The petrographic investigations were carried out by a Nikon YS2-T polarizing microscope and an AMRAY 1830 I/T6 scanning electron microscope at the Department of Petrology and Geochemistry of the Eötvös Loránd University. Whole-rock chemical compositions were determined by ICP-MS (AcmeLabs Ltd., Vancouver, Canada; http://acmelab.com/). In situ major element analyses of the mineral phases were performed using a CAMECA SX100 electron microprobe equipped with four WDS and an EDS at the University of Vienna, Department of Lithospheric Research. Operating conditions were $15 \mathrm{kV}$ accelerating voltage, 20 $\mathrm{nA}$ beam current, focused beam diameter and ZAF correction procedure for data reduction. Calibration was based on the following standards: albite $(\mathrm{Na}, \mathrm{Si}, \mathrm{Al})$, olivine $(\mathrm{Mg})$, almandine $(\mathrm{Fe})$, wollastonite $(\mathrm{Ca})$, rutile $(\mathrm{Ti})$, spessartine $(\mathrm{Mn})$, orthoclase (K), Mg-chromite ( $\mathrm{Cr}$ ) and Ni-oxide $(\mathrm{Ni})$.

\section{Petrography}

The studied rocks were selected from a large sample collection. They are fairly uniform from a petrographic point of view: the texture is medium to highly vesicular and they are relatively crystal-rich (up to 50 vol\% crystals; Fig. 3). The crystals have variable shapes and sizes. Approximately the half of the rock consists of anhedral crystals which have resorbed rims and their sizes vary from 0.2 to $5 \mathrm{~mm}$. They are olivine, clinopyroxene, orthopyroxene and spinel (Fig. 4).

Fig. 3

Textural features and modal mineralogy of the studied alkaline basalt (BSE image) (ol - olivine, cpx - clinopyroxene; opx - orthopyroxene; sp - spinel)

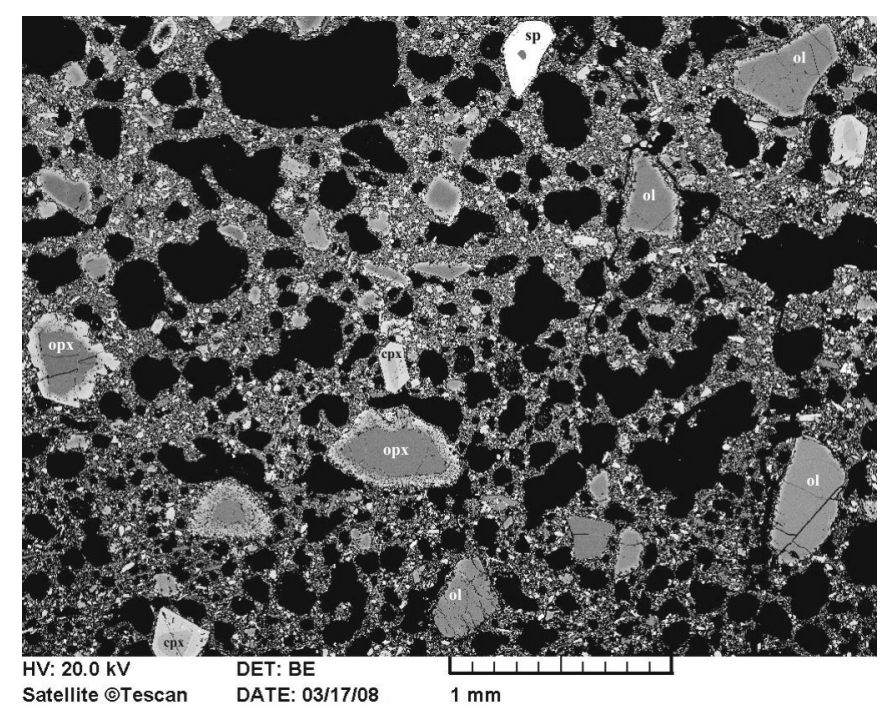




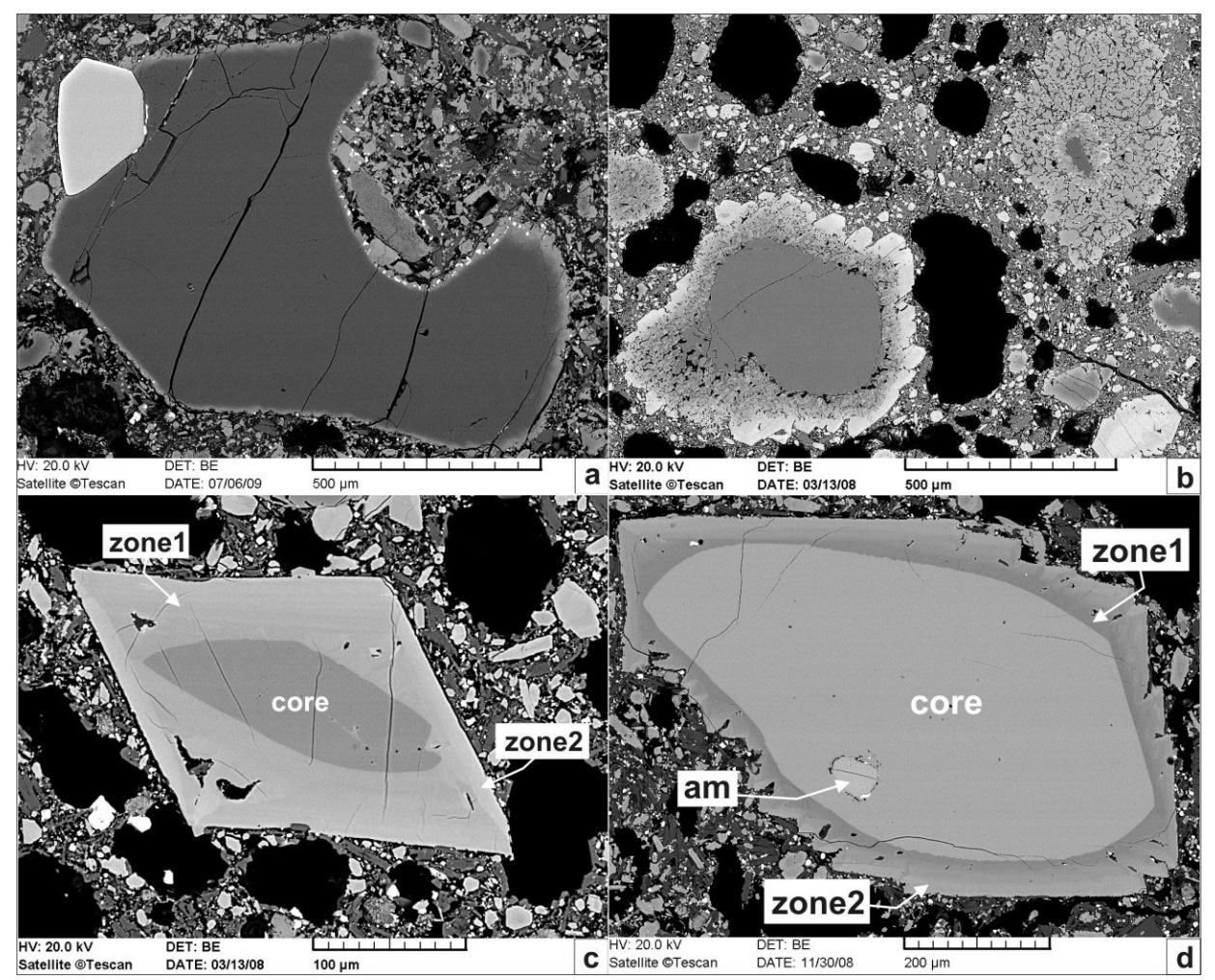

Fig. 4

a) Olivine xenocryst with a light brown spinel xenocryst; b) Orthopyroxene xenocryst with thin reaction rim overgrown by a zoned clinopyroxene phenocryst (left); orthopyroxene xenocryst with thick reaction rim in which the minerals are coarser outward (right); c) Strongly resorbed colourless clinopyroxene xenocryst (core) mantled by zoned clinopyroxene phenocryst (zone1 and zone2); d) Rounded light green clinopyroxene xenocryst (core) overgrown by zoned clinopyroxene phenocryst (zone1 and zone2); the xenocryst contains an amphibole (BSE images)

The euhedral to subhedral phenocrysts are olivine, clinopyroxene and occasional plagioclase. Olivine, clinopyroxene, plagioclase and Fe-Ti-oxides appear as microphenocrysts $(<150 \mu \mathrm{m})$. The groundmass consists of microlithic plagioclase, olivine, clinopyroxene, Fe-Ti-oxides and glass (tachylite).

Olivine is the most abundant mineral phase (approximately $40-50 \mathrm{vol} \%$ of crystals). The grains often have rounded and embayed margins (Fig. 4a), which sometimes show spongy texture. They usually have very thin rims and frequently contain rounded spinel inclusions $(\sim 50-250 \mu \mathrm{m})$ with different colours (brownish-green, light brown, dark brown and their transitions). These all suggest that they are xenocrysts. Olivine phenocrysts $(150-250 \mu \mathrm{m})$ are euhedral to subhedral and show normal zoning. Their outermost margin is usually iddingsitized. Light green to light brown, euhedral to subhedral Cr-spinel 
inclusions $(\sim 5-15 \mu \mathrm{m})$ can be occasionally observed, mainly in the margins (Fig. $9 b)$. Titanomagnetite inclusions are also present.

Orthopyroxene has a reaction rim of variable thickness (Fig. 4b). It consists of olivine, clinopyroxene, glass and sometimes spinel. In some cases the size of these crystals increases outward from the margin of the orthopyroxene grain. The reaction rim is frequently overgrown by zoned clinopyroxene. This reaction rim implies that the orthopyroxene grains are xenocrysts. Sometimes they also contain brownish spinel inclusions, like the olivine xenocrysts.

Clinopyroxene forms euhedral to subhedral crystals showing variable zoning patterns. Most of the phenocrysts have an anhedral colourless core, but some of them have a light or dark green core with a rounded margin (Fig. 4c, d). The colourless cores occasionally contain brown, rounded spinel inclusions like in the olivine xenocrysts, while in one of the light green cores a greenish-brown, pleochroic amphibole inclusion $(\sim 60 \mu \mathrm{m})$ was recognized. These features infer that the rounded colourless and green cores of clinopyroxenes are xenocrysts as well. Zoned phenocrysts can be also found as overgrowth on the reaction rim of orthopyroxene xenocrysts.

Spinel can be found as inclusion in various minerals and also as xenocryst in the matrix. It shows different colours such as brownish-green, light brown, dark brown and their transitions. The xenocrystic spinels often have a black rim of variable thicknesses.

In addition to the abundant xenocrysts, the basalt bombs include numerous fragments of peridotite xenoliths. They are spinel-peridotites, which occasionally contain a great number of anhedral, yellowish-brown and pleochroic amphiboles. Apatite grains can be also found in the xenoliths, but they are rare.

\section{Whole-rock geochemistry}

Whole-rock analysis was performed for a representative scoriaceous bomb and a massive lava fragment. The major element compositions (Table 1) are similar to the composition of the other alkaline basalt in the Bakony-Balaton Highland Volcanic Field (Embey-Isztin et al. 1993), apart from the $\mathrm{MgO}$ content. The studied alkaline basalt is extremely rich in $\mathrm{MgO}$ (scoria: $13.41 \mathrm{wt} \%$; lava: 14.21 wt\%) similarly to that described only in the Bondoró locality (Fig. 1b) so far $(\mathrm{MgO}=13.9 \mathrm{wt} \%$; Jugovics, 1976; Embey-Isztin et al. 1993). This is consistent with the abundance of xenocrystic magnesian minerals, such as olivine and orthopyroxene. According to their total alkalis relative to silica content the studied samples are basanites (Le Bas et al. 1986).

The trace element composition (Table 2) of the two samples is identical, suggesting that they represent eruptions of the same magma. However, differences can be seen in the $\mathrm{Cs}, \mathrm{Rb}$ and $\mathrm{K}$ contents (Fig. 5). The strongly negative K-anomaly characterized by the scoriaceous bomb does not correlate with the variation of the $\mathrm{Rb}$ and $\mathrm{Cs}$ concentrations and may thus be a secondary 
Table 1

Major element analyses (in wt $\%$; total $\mathrm{Fe}$ is in the form of $\mathrm{Fe}_{2} \mathrm{O}_{3}{ }^{*}$ ) of the studied samples from the Füzes-tó scoria cone

\begin{tabular}{ccc}
\hline & FT-9 & FT-10 \\
\hline $\mathrm{SiO}_{2}$ & 45.40 & 43.84 \\
$\mathrm{TiO}_{2}$ & 1.67 & 1.59 \\
$\mathrm{Al}_{2} \mathrm{O}_{3}$ & 13.60 & 13.13 \\
$\mathrm{Fe}_{2} \mathrm{O}_{3}{ }^{*}$ & 9.49 & 9.41 \\
$\mathrm{MnO}$ & 0.16 & 0.16 \\
$\mathrm{MgO}$ & 13.41 & 14.21 \\
$\mathrm{CaO}$ & 8.01 & 8.89 \\
$\mathrm{Na}{ }_{2} \mathrm{O}$ & 3.81 & 3.46 \\
$\mathrm{~K} 2 \mathrm{O}$ & 1.03 & 1.87 \\
$\mathrm{P}_{2} \mathrm{O}_{5}$ & 0.72 & 0.67 \\
$\mathrm{LOI}$ & 2.10 & 2.10 \\
\hline Total & 99.50 & 99.50 \\
\hline
\end{tabular}

FT-9=scoria, FT-10=lava

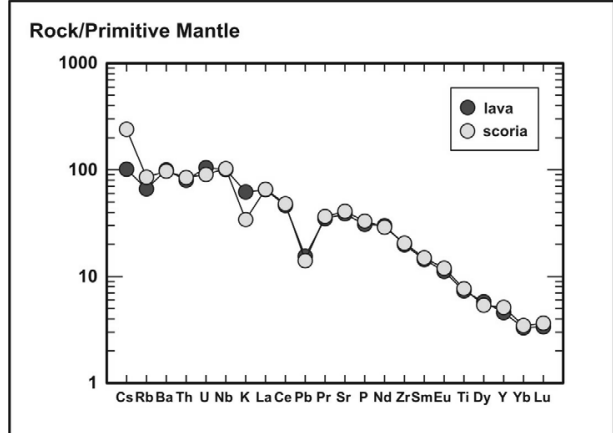

Fig. 5

Primitive mantle normalized (McDonough and Sun 1995) trace element variation diagram of the studied rocks
Table 2

Trace element concentrations (in ppm) of the studied samples from the Füzes-tó scoria cone

\begin{tabular}{lcc}
\hline & FT-9 & FT-10 \\
\hline $\mathrm{Ni}$ & 513 & 564 \\
$\mathrm{Cr}$ & 595 & 657 \\
$\mathrm{~V}$ & 154 & 152 \\
$\mathrm{Sc}$ & 18 & 18 \\
$\mathrm{Co}$ & 45.8 & 47.1 \\
$\mathrm{Cs}$ & 1.9 & 0.8 \\
$\mathrm{Rb}$ & 54.2 & 42.2 \\
$\mathrm{Ba}$ & 679 & 697 \\
$\mathrm{~Pb}$ & 1 & 1.1 \\
$\mathrm{Sr}$ & 864.3 & 819.4 \\
$\mathrm{Zr}$ & 230.1 & 222.2 \\
$\mathrm{Nb}$ & 73.1 & 71.7 \\
$\mathrm{Y}$ & 23.3 & 20.9 \\
$\mathrm{Th}$ & 7.2 & 6.8 \\
$\mathrm{La}$ & 45.2 & 44.8 \\
$\mathrm{Ce}$ & 85.4 & 82.4 \\
$\mathrm{Pr}$ & 10.1 & 9.59 \\
$\mathrm{Nd}$ & 39.2 & 40.7 \\
$\mathrm{Sm}$ & 6.65 & 6.41 \\
$\mathrm{Eu}$ & 2.01 & 1.88 \\
$\mathrm{Gd}$ & 5.47 & 5.15 \\
$\mathrm{~Tb}$ & 0.67 & 0.65 \\
$\mathrm{Dy}$ & 3.96 & 4.26 \\
$\mathrm{Ho}$ & 0.76 & 0.74 \\
$\mathrm{Er}$ & 1.95 & 1.94 \\
$\mathrm{Yb}$ & 1.71 & 1.63 \\
$\mathrm{Lu}$ & 0.27 & 0.25 \\
$\mathrm{Hf}$ & 5.3 & 5.4 \\
$\mathrm{Ta}$ & 4.4 & 4.1 \\
$\mathrm{U}$ & 1.9 & 2.2 \\
\hline
\end{tabular}


feature reflecting selective K-depletion during or after the eruption. The high $\mathrm{MgO}$ content of the bulk rocks is accompanied by extreme enrichment in $\mathrm{Ni}$ and Cr contents $(\mathrm{Ni}=513-564 \mathrm{ppm} ; \mathrm{Cr}=595-657 \mathrm{ppm})$. This can be explained by accumulation of magnesian minerals, mostly as xenocrysts.

\section{Mineral chemistry}

\section{Olivine}

Olivine has a wide range of Fo content (Table 3 ). The xenocrysts are typically magnesian-rich $\left(\mathrm{Fo}_{89-91}\right)$. They have a thin rim with lower forsterite values $\left(\mathrm{Fo}_{79-85}\right)$. The phenocrysts show normal zoning with magnesian cores $\left(\mathrm{Fo}_{87-89}\right)$, whereas the rims are in the range of $\mathrm{Fo}_{74-84}$. The composition of the olivine grains in the reaction rim around orthopyroxene xenocrysts is from $\mathrm{Fo}_{81}$ to $\mathrm{Fo}_{87}$.

The $\mathrm{CaO}$ contents of olivines are plotted against the Fo values in Fig. 6, which shows a negative correlation. Xenocrysts contain the least $\mathrm{CaO}(0.03-0.11 \mathrm{wt} \%)$, while their thin rims have higher $\mathrm{CaO}$ content $(0.21-0.30 \mathrm{wt} \%)$. The phenocrysts have an increasing $\mathrm{CaO}$ content with decreasing Fo from their cores $(0.14-0.25$ $\mathrm{wt} \%)$ to the rims $(0.23-0.37 \mathrm{wt} \%)$. The $\mathrm{CaO}$ content of the olivines in the reaction rim of orthopyroxene xenocrysts is $0.18-0.32 \mathrm{wt} \%$. The $\mathrm{NiO}$ contents correlate positively with Fo. The xenocrysts have a high $\mathrm{NiO}$ content $(0.36-0.42 \mathrm{wt} \%)$, whereas their thin rim contains much less $\mathrm{NiO}(0.11-0.29 \mathrm{wt} \%)$. The phenocrysts have a decreasing $\mathrm{NiO}$ content from their cores $(0.14-0.31 \mathrm{wt} \%)$ to the rims $(0.10-0.23 \mathrm{wt} \%)$. The $\mathrm{NiO}$ content of olivines in the reaction rim of orthopyroxene xenocrysts ranges from 0.12 to $0.20 \mathrm{wt} \%$. The composition of the thin rim of the olivine xenocrysts is similar to the composition of the phenocryst rims.

Fig. 6

Relationship between the Fo and $\mathrm{CaO}$ content of the studied olivines

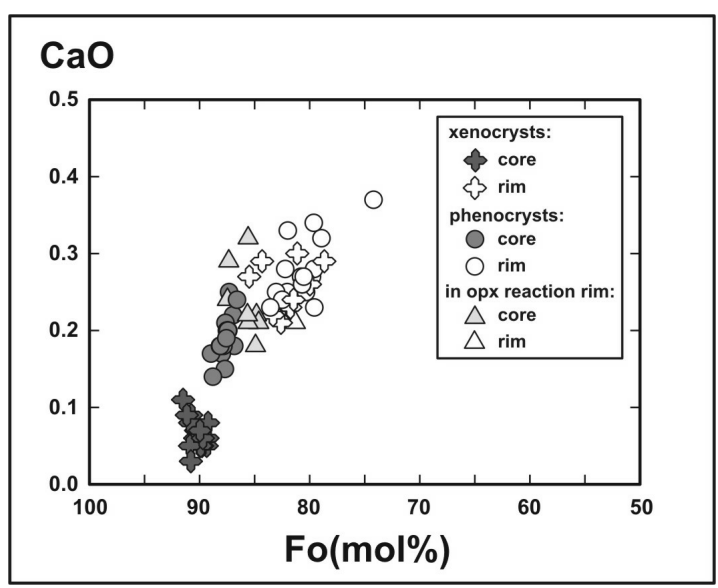


Table 3

Representative compositions of the studied olivines

\begin{tabular}{|c|c|c|c|c|c|c|c|c|}
\hline & \multicolumn{4}{|c|}{ xenocryst } & \multicolumn{4}{|c|}{ thin rim of xenocryst } \\
\hline & ol1_core & ol2_core & ol3_core & ol4_core & ol1_rim & ol2_rim & ol3_rim & ol4_rim \\
\hline $\mathrm{SiO}_{2}$ & 40.88 & 41.20 & 40.78 & 40.88 & 38.96 & 39.91 & 38.36 & 39.40 \\
\hline $\mathrm{MgO}$ & 49.34 & 49.65 & 50.07 & 48.70 & 41.26 & 45.40 & 40.59 & 42.82 \\
\hline $\mathrm{FeO}$ & 9.34 & 8.90 & 8.30 & 10.43 & 18.32 & 13.74 & 19.62 & 16.92 \\
\hline $\mathrm{MnO}$ & 0.15 & 0.12 & 0.13 & 0.16 & 0.47 & 0.26 & 0.59 & 0.50 \\
\hline $\mathrm{CaO}$ & 0.08 & 0.05 & 0.11 & 0.06 & 0.26 & 0.27 & 0.29 & 0.23 \\
\hline $\mathrm{NiO}$ & 0.42 & 0.41 & 0.36 & 0.41 & 0.18 & 0.29 & 0.11 & 0.21 \\
\hline $\mathrm{Cr}_{2} \mathrm{O}_{3}$ & 0.00 & 0.09 & 0.06 & 0.00 & 0.01 & 0.00 & 0.00 & 0.03 \\
\hline Total & 100.23 & 100.42 & 99.88 & 100.66 & 99.49 & 99.89 & 99.68 & 100.11 \\
\hline $\mathrm{Fo}(\mathrm{mol} \%)$ & 90.4 & 90.9 & 91.5 & 89.3 & 80.1 & 85.5 & 78.7 & 81.9 \\
\hline \multicolumn{9}{|c|}{ ol=olivine; opx=orthopyroxene } \\
\hline & \multicolumn{4}{|c|}{ phenocryst core } & \multicolumn{4}{|c|}{ phenocryst rim } \\
\hline & ol5_core & ol6_core & ol7_core & ol8_core & ol5_rim & ol6_rim & ol7_rim & ol8_rim \\
\hline $\mathrm{SiO}_{2}$ & 40.12 & 40.62 & 40.54 & 40.05 & 38.65 & 39.35 & 38.99 & 38.67 \\
\hline $\mathrm{MgO}$ & 48.17 & 47.40 & 47.22 & 47.80 & 42.28 & 42.31 & 41.45 & 41.46 \\
\hline $\mathrm{FeO}$ & 10.84 & 11.54 & 11.65 & 11.45 & 18.05 & 16.51 & 17.58 & 18.90 \\
\hline $\mathrm{MnO}$ & 0.17 & 0.17 & 0.18 & 0.14 & 0.48 & 0.50 & 0.57 & 0.54 \\
\hline $\mathrm{CaO}$ & 0.14 & 0.17 & 0.18 & 0.18 & 0.26 & 0.25 & 0.27 & 0.34 \\
\hline $\mathrm{NiO}$ & 0.27 & 0.29 & 0.20 & 0.23 & 0.16 & 0.18 & 0.16 & 0.15 \\
\hline $\mathrm{Cr}_{2} \mathrm{O}_{3}$ & 0.01 & 0.02 & 0.04 & 0.04 & 0.03 & 0.00 & 0.02 & 0.03 \\
\hline Total & 99.74 & 100.23 & 100.04 & 99.95 & 99.99 & 99.11 & 99.07 & 100.11 \\
\hline \multirow[t]{3}{*}{$\mathrm{Fo}(\mathrm{mol} \%)$} & 88.8 & 88.0 & 87.8 & 88.1 & 80.7 & 82.0 & 80.8 & 79.6 \\
\hline & \multicolumn{2}{|c|}{$\begin{array}{l}\text { in the reaction rim of } \\
\text { opx }\end{array}$} & & & & & & \\
\hline & ol9_core & ol9_rim & & & & & & \\
\hline $\mathrm{SiO}_{2}$ & 39.58 & 38.74 & & & & & & \\
\hline $\mathrm{MgO}$ & 46.41 & 42.57 & & & & & & \\
\hline $\mathrm{FeO}$ & 11.99 & 17.46 & & & & & & \\
\hline $\mathrm{MnO}$ & 0.23 & 0.40 & & & & & & \\
\hline $\mathrm{CaO}$ & 0.29 & 0.21 & & & & & & \\
\hline $\mathrm{NiO}$ & 0.19 & 0.12 & & & & & & \\
\hline $\mathrm{Cr}_{2} \mathrm{O}_{3}$ & 0.10 & 0.04 & & & & & & \\
\hline Total & 98.87 & 99.56 & & & & & & \\
\hline $\mathrm{Fo}(\mathrm{mol} \%)$ & 87.3 & 81.3 & & & & & & \\
\hline
\end{tabular}

\section{Orthopyroxene}

Orthopyroxene xenocrysts are enstatites and ferroan-enstatites (Morimoto 1988). They have high Mg-numbers (Fe as total $\left.\mathrm{Fe}_{\text {tot }}\right)$, ranging between $0.88-0.92$ (Table 4), typical of mantle minerals. Reaction rim around the orthopyroxene 
consists of olivine, clinopyroxene, $\mathrm{Si}$ rich glass and spinel, the composition of which depends on their place in the rim.

\section{Clinopyroxene}

Representative clinopyroxene analyses are presented in Table 5 . In the phenocrysts the colourless cores are diopsides (Morimoto 1988) and they are characterized by high Mg-number $\left(0.90-0.92 ; \mathrm{Mg} /\left(\mathrm{Mg}+\mathrm{Fe}_{\text {tot }}\right)\right)$. Most of the green cores are ferroan-diopside, but one of them is Mg-hedenbergite (Morimoto 1988). The green cores are typically Fe-rich (11.02-17.18 wt\% $\mathrm{FeO})$. The rims around the xenocrystic cores are ferroan-diopside, similar to the composition of the clinopyroxene phenocrysts and microphenocrysts. The clinopyroxene grains in the reaction rim of the orthopyroxene xenocrysts are (Mg-rich) augites and ferroan-diopsides.

The zoned pheno- and microphenocrysts have an increasing $\mathrm{Al}$ and Ti content from their zone1 to zone2 (Fig. 7a). Compositions of the colourless and green anhedral cores of the crystals do not fit in the trend formed by the pheno- and microphenocrysts, and therefore they can be regarded as xenocrysts. The green

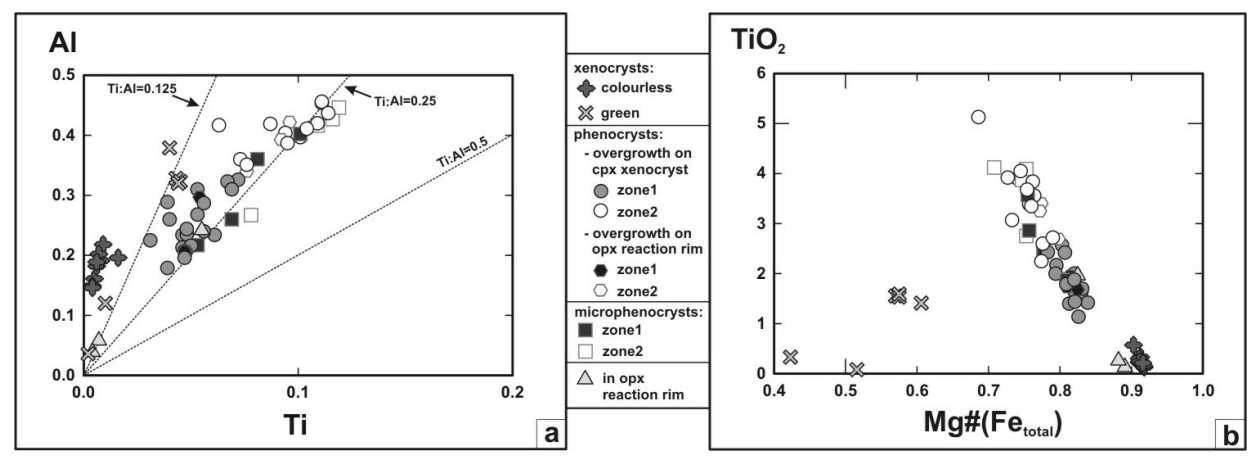

Fig. 7

a) Variation of $\mathrm{Ti}$ and $\mathrm{Al}$ in the studied clinopyroxenes; b) Plots of $\mathrm{Mg}$-number vs. $\mathrm{TiO}_{2}$ in the studied clinopyroxenes

Representative compositions of the studied orthopyroxenes

\begin{tabular}{|c|c|c|c|c|}
\hline & opx1 & opx2 & opx3 & opx4 \\
\hline $\mathrm{SiO}_{2}$ & 55.69 & 54.67 & 55.47 & 53.53 \\
\hline $\mathrm{TiO}_{2}$ & 0.05 & 0.07 & 0.04 & 0.12 \\
\hline $\mathrm{Al}_{2} \mathrm{O}_{3}$ & 3.54 & 4.02 & 2.39 & 4.60 \\
\hline $\mathrm{Cr}_{2} \mathrm{O}_{3}$ & 0.41 & 0.39 & 0.42 & 0.18 \\
\hline $\mathrm{FeO}$ & 5.86 & 6.41 & 5.61 & 7.81 \\
\hline $\mathrm{MnO}$ & 0.12 & 0.16 & 0.13 & 0.17 \\
\hline $\mathrm{NiO}$ & 0.10 & 0.07 & 0.09 & 0.09 \\
\hline $\mathrm{MgO}$ & 33.68 & 33.03 & 34.69 & 32.38 \\
\hline $\mathrm{CaO}$ & 0.65 & 0.75 & 0.65 & 0.73 \\
\hline Total & 100.13 & 99.58 & 99.49 & 99.61 \\
\hline$\left(\mathrm{Fe}^{\mathrm{tot}}\right)$ & 0.91 & 0.90 & 0.92 & 0.88 \\
\hline En & 89.81 & 88.66 & 90.40 & 86.61 \\
\hline Wo & 1.25 & 1.44 & 1.21 & 1.41 \\
\hline Fs & 8.95 & 9.90 & 8.39 & 11.99 \\
\hline
\end{tabular}


Table 5

Representative compositions of the studied clinopyroxenes.

$\mathrm{cpx}=$ clinopyroxene; opx = orthopyroxene; $\mathrm{ph}=$ phenocryst; $\mathrm{Mg} \#\left(\mathrm{Fe}_{\mathrm{tot}}\right)=\mathrm{Mg} /\left(\mathrm{Mg}+\mathrm{Fe}_{\text {tot }}\right)$

\begin{tabular}{|c|c|c|c|c|c|c|}
\hline & \multicolumn{2}{|c|}{ colourless xenocryst } & \multicolumn{4}{|c|}{ phenocryst:overgrowth on colourless xenocryst } \\
\hline & cpx1_core & cpx2_core & cpx1_zone1 & cpx1_zone2 & cpx2_zone1 & cpx2_zone2 \\
\hline $\mathrm{SiO}_{2}$ & 52.62 & 51.29 & 47.53 & 40.52 & 46.11 & 42.11 \\
\hline $\mathrm{TiO}_{2}$ & 0.13 & 0.30 & 1.89 & 5.13 & 2.43 & 3.90 \\
\hline $\mathrm{Al}_{2} \mathrm{O}_{3}$ & 3.48 & 4.44 & 7.09 & 11.43 & 7.01 & 10.19 \\
\hline $\mathrm{Cr}_{2} \mathrm{O}_{3}$ & 0.42 & 0.52 & 0.83 & 0.06 & 0.06 & 0.20 \\
\hline $\mathrm{FeO}$ & 2.70 & 2.91 & 5.65 & 8.16 & 6.55 & 7.13 \\
\hline $\mathrm{MnO}$ & 0.09 & 0.06 & 0.13 & 0.14 & 0.13 & 0.11 \\
\hline $\mathrm{MgO}$ & 17.02 & 16.70 & 13.39 & 10.01 & 13.25 & 11.42 \\
\hline $\mathrm{CaO}$ & 23.34 & 22.35 & 21.96 & 22.66 & 22.43 & 22.38 \\
\hline $\mathrm{Na}_{2} \mathrm{O}$ & 0.25 & 0.53 & 0.78 & 0.59 & 0.45 & 0.55 \\
\hline Total & 100.14 & 99.12 & 99.28 & 98.71 & 98.43 & 98.00 \\
\hline $\mathrm{Mg} \#\left(\mathrm{Fe}^{\mathrm{tot}}\right)$ & 0.92 & 0.91 & 0.81 & 0.69 & 0.78 & 0.74 \\
\hline En & 48.13 & 48.50 & 41.30 & 32.32 & 40.01 & 36.18 \\
\hline Wo & 47.45 & 46.66 & 48.70 & 52.63 & 48.68 & 50.95 \\
\hline \multirow[t]{3}{*}{ Fs } & 4.43 & 4.84 & 10.00 & 15.05 & 11.31 & 12.86 \\
\hline & \multicolumn{6}{|c|}{ green xenocryst } \\
\hline & cpx3_core & cpx4_core & cpx5_core & cpx6_core & cpx7_core & cpx8_core \\
\hline $\mathrm{SiO}_{2}$ & 47.58 & 50.96 & 46.86 & 48.44 & 47.53 & 47.13 \\
\hline $\mathrm{TiO}_{2}$ & 1.55 & 0.08 & 1.41 & 0.33 & 1.53 & 1.59 \\
\hline $\mathrm{Al}_{2} \mathrm{O}_{3}$ & 7.19 & 0.80 & 8.57 & 2.59 & 7.38 & 7.24 \\
\hline $\mathrm{Cr}_{2} \mathrm{O}_{3}$ & 0.01 & 0.05 & 0.03 & 0.08 & 0.00 & 0.02 \\
\hline $\mathrm{FeO}$ & 11.84 & 14.73 & 11.02 & 17.18 & 11.80 & 11.69 \\
\hline $\mathrm{MnO}$ & 0.26 & 0.33 & 0.25 & 0.29 & 0.25 & 0.26 \\
\hline $\mathrm{MgO}$ & 8.82 & 8.82 & 9.50 & 7.07 & 8.94 & 8.87 \\
\hline $\mathrm{CaO}$ & 19.90 & 23.58 & 20.02 & 22.23 & 19.88 & 19.95 \\
\hline $\mathrm{Na}_{2} \mathrm{O}$ & 2.11 & 0.16 & 1.71 & 0.34 & 2.18 & 2.08 \\
\hline Total & 99.27 & 99.51 & 99.37 & 98.69 & 99.49 & 98.83 \\
\hline $\mathrm{Mg} \#\left(\mathrm{Fe}^{\mathrm{tot}}\right)$ & 0.57 & 0.52 & 0.61 & 0.42 & 0.57 & 0.58 \\
\hline En & 29.48 & 25.77 & 31.46 & 21.52 & 29.81 & 29.66 \\
\hline Wo & 47.82 & 49.53 & 47.63 & 48.64 & 47.65 & 47.94 \\
\hline Fs & 22.70 & 24.70 & 20.92 & 29.84 & 22.54 & 22.41 \\
\hline
\end{tabular}

xenocrysts have variable $\mathrm{Al}$ content and are separated from the colourless ones. The clinopyroxenes in the reaction rims of the orthopyroxene xenocrysts are characterized by varied $\mathrm{Al}$ and $\mathrm{Ti}$ content.

The $\mathrm{Mg}$-number- $\mathrm{TiO}_{2}$ diagram (Fig. $7 \mathrm{~b}$ ) also separates the xenocrystic clinopyroxene from the magmatic ones. Zoned pheno- and microphenocrysts have an increasing Ti content with decreasing $\mathrm{Mg \#}\left(\mathrm{Fe}_{\text {tot }}\right)$ from their zone1 to zone2. The variable compositions of the clinopyroxenes in the reaction rims of the orthopyroxenes can also be observed in Fig. $7 \mathrm{~b}$. 
Table 5 (cont.)

\begin{tabular}{|c|c|c|c|c|c|c|c|}
\hline & \multicolumn{7}{|c|}{ phenocryst:overgrowth on green xenocryst } \\
\hline & cpx3_zone1 & cpx3_zone2 & cpx4_zone1 & cpx4_zone2 & cpx5_zone1 & cpx5_zone2 & cpx6_zone1 \\
\hline $\mathrm{SiO}_{2}$ & 47.78 & 45.02 & 49.46 & 44.07 & 48.71 & 43.05 & 47.93 \\
\hline $\mathrm{TiO}_{2}$ & 2.00 & 3.39 & 1.68 & 3.68 & 1.40 & 3.92 & 1.70 \\
\hline $\mathrm{Al}_{2} \mathrm{O}_{3}$ & 6.58 & 8.79 & 4.50 & 9.31 & 6.63 & 10.23 & 5.56 \\
\hline $\mathrm{Cr}_{2} \mathrm{O}_{3}$ & 0.48 & 0.20 & 0.22 & 0.17 & 0.77 & 0.05 & 0.79 \\
\hline $\mathrm{FeO}$ & 5.39 & 6.84 & 5.62 & 6.83 & 5.71 & 7.38 & 5.13 \\
\hline $\mathrm{MnO}$ & 0.10 & 0.13 & 0.09 & 0.12 & 0.14 & 0.12 & 0.12 \\
\hline $\mathrm{MgO}$ & 13.74 & 11.97 & 14.50 & 11.73 & 13.90 & 11.03 & 14.19 \\
\hline $\mathrm{CaO}$ & 23.07 & 22.62 & 22.86 & 22.64 & 21.15 & 22.78 & 22.85 \\
\hline $\mathrm{Na}_{2} \mathrm{O}$ & 0.52 & 0.51 & 0.35 & 0.54 & 0.93 & 0.58 & 0.51 \\
\hline Total & 99.67 & 99.50 & 99.31 & 99.12 & 99.35 & 99.13 & 98.94 \\
\hline $\mathrm{Mg} \#\left(\mathrm{Fe}^{\mathrm{tot}}\right)$ & 0.82 & 0.76 & 0.82 & 0.75 & 0.81 & 0.73 & 0.83 \\
\hline En & 41.13 & 37.24 & 42.48 & 36.77 & 42.92 & 34.89 & 42.27 \\
\hline Wo & 49.64 & 50.59 & 48.14 & 51.01 & 46.94 & 51.79 & 48.94 \\
\hline \multirow[t]{3}{*}{$\mathrm{Fs}$} & 9.22 & 12.17 & 9.39 & 12.23 & 10.14 & 13.32 & 8.78 \\
\hline & \multicolumn{5}{|c|}{ phenocryst:overgrowth on green xenocryst } & \multicolumn{2}{|c|}{$\begin{array}{c}\text { ph:overgrowth on opx } \\
\text { reaction rim }\end{array}$} \\
\hline & cpx6_zone2 & cpx7_zone1 & cpx7_zone2 & cpx8_zone1 & cpx8_zone2 & cpx9_zone1 & cpx9_zone2 \\
\hline $\mathrm{SiO}_{2}$ & 42.91 & 49.38 & 43.52 & 48.47 & 44.42 & 49.06 & 44.13 \\
\hline $\mathrm{TiO}_{2}$ & 3.84 & 1.66 & 4.05 & 2.00 & 3.35 & 1.73 & 3.40 \\
\hline $\mathrm{Al}_{2} \mathrm{O}_{3}$ & 9.43 & 4.91 & 9.91 & 5.51 & 9.12 & 4.70 & 9.58 \\
\hline $\mathrm{Cr}_{2} \mathrm{O}_{3}$ & 0.36 & 0.29 & 0.15 & 0.20 & 0.41 & 0.26 & 0.62 \\
\hline $\mathrm{FeO}$ & 6.60 & 5.65 & 7.02 & 6.45 & 6.67 & 6.00 & 6.24 \\
\hline $\mathrm{MnO}$ & 0.13 & 0.13 & 0.15 & 0.16 & 0.12 & 0.14 & 0.11 \\
\hline $\mathrm{MgO}$ & 11.88 & 14.47 & 11.50 & 13.97 & 11.85 & 14.36 & 12.01 \\
\hline $\mathrm{CaO}$ & 22.61 & 22.83 & 22.65 & 22.44 & 22.46 & 22.65 & 22.69 \\
\hline $\mathrm{Na}_{2} \mathrm{O}$ & 0.54 & 0.47 & 0.52 & 0.43 & 0.56 & 0.37 & 0.53 \\
\hline Total & 98.31 & 99.80 & 99.50 & 99.64 & 98.97 & 99.29 & 99.32 \\
\hline $\mathrm{Mg} \#\left(\mathrm{Fe}^{\mathrm{tot}}\right)$ & 0.76 & 0.82 & 0.74 & 0.79 & 0.76 & 0.81 & 0.77 \\
\hline En & 37.23 & 42.41 & 36.15 & 41.32 & 37.27 & 42.13 & 37.67 \\
\hline Wo & 50.94 & 48.08 & 51.19 & 47.71 & 50.76 & 47.76 & 51.16 \\
\hline \multirow[t]{3}{*}{ Fs } & 11.83 & 9.51 & 12.66 & 10.97 & 11.97 & 10.11 & 11.18 \\
\hline & \multicolumn{2}{|c|}{ microphenocryst } & \multicolumn{2}{|c|}{ in the reaction rim of opx } & & & \\
\hline & cpx10_zone1 & cpx10_zone2 & $\mathrm{cpx} 11$ & $\operatorname{cpx} 12$ & & & \\
\hline $\mathrm{SiO}_{2}$ & 47.31 & 43.57 & 48.00 & 53.12 & & & \\
\hline $\mathrm{TiO}_{2}$ & 2.44 & 3.87 & 1.96 & 0.26 & & & \\
\hline $\mathrm{Al}_{2} \mathrm{O}_{3}$ & 5.91 & 9.40 & 5.54 & 1.31 & & & \\
\hline $\mathrm{Cr}_{2} \mathrm{O}_{3}$ & 0.04 & 0.22 & 0.35 & 0.67 & & & \\
\hline $\mathrm{FeO}$ & 6.82 & 6.99 & 5.43 & 4.32 & & & \\
\hline $\mathrm{MnO}$ & 0.12 & 0.15 & 0.11 & 0.15 & & & \\
\hline $\mathrm{MgO}$ & 13.33 & 11.61 & 14.33 & 18.14 & & & \\
\hline $\mathrm{CaO}$ & 22.67 & 22.78 & 23.04 & 20.46 & & & \\
\hline $\mathrm{Na}_{2} \mathrm{O}$ & 0.46 & 0.56 & 0.38 & 0.69 & & & \\
\hline Total & 99.13 & 99.18 & 99.14 & 99.59 & & & \\
\hline $\mathrm{Mg} \#\left(\mathrm{Fe}^{\mathrm{tot}}\right)$ & 0.78 & 0.75 & 0.82 & 0.88 & & & \\
\hline En & 39.77 & 36.29 & 42.16 & 51.30 & & & \\
\hline
\end{tabular}


Spinel

Representative spinel analyses are shown in Table 6. The spinel xenocrysts have a variable composition according to their colour. The brownish-green spinels are characterized by the highest $\mathrm{Mg}$-number $\left(0.74-0.76\right.$, Fe as total $\left.\mathrm{Fe}_{\text {tot }}\right)$ and $\mathrm{Al}_{2} \mathrm{O}_{3}$ content (53.74-58.03 wt\%) and the lowest $\mathrm{Cr}_{2} \mathrm{O}_{3}$ content (9.19-12.33 $\left.{ }_{w t} \%\right)$ and $\mathrm{Cr}$-number $(9.63-13.34 ; 100 * \mathrm{Cr} /(\mathrm{Cr}+\mathrm{Al}))$. The dark brown grains have the least $\mathrm{Mg}$-number $(0.58-0.68)$ and $\mathrm{Al}_{2} \mathrm{O}_{3}(27.31-36.41 \mathrm{wt} \%)$ and are the richest in $\mathrm{Cr}$ (30.38-37.69 wt\% $\mathrm{Cr}_{2} \mathrm{O}_{3}$ and 36.00-48.07 Cr-number). The light brown spinels have a transitional composition between the two others.

The spinel inclusions in olivine phenocrysts have high $\mathrm{Al}_{2} \mathrm{O}_{3}$ content (26.37-40.86 wt\%) and relatively low $\mathrm{Cr}_{2} \mathrm{O}_{3}$ content (17.21-23.17 wt\%), so their Cr-number is characteristically low (22.10-34.78). Their $\mathrm{TiO}_{2}$ contents range from 0.64 to $2.17 \mathrm{wt} \%$. Spinels in the reaction rim of the orthopyroxene xenocrysts are poor in $\mathrm{Al}\left(2.75-5.63 \mathrm{wt} \% \mathrm{Al}_{2} \mathrm{O}_{3}\right)$ and rich in $\mathrm{Ti}\left(2.87-6.52 \mathrm{wt} \% \mathrm{TiO}_{2}\right)$ and $\mathrm{Fe}$ (43.75-58.08 wt\% FeO).

\section{Other minerals}

Plagioclase microphenocrysts have fairly uniform, labradoritic compositions $\left(\mathrm{An}_{62}-\mathrm{An}_{65}\right)$. The Fe-Ti-oxides are magnetites having high $\mathrm{FeO}(80.42-81.25 \mathrm{wt} \%)$ and low $\mathrm{TiO}_{2}$ content $(0.35-0.53 \mathrm{wt} \%)$. Their ulvospinel (Usp) contents are very low (0.01-0.02 mol\%). Titano-magnetites contain $\mathrm{TiO}_{2}$ of $5.54-16.24 \mathrm{wt} \%$, while their FeO content are 64.04-78.46 wt\%, and they have variable ulvospinel (Usp) contents $(0.15-0.68 \mathrm{~mol} \%)$. These compositions suggest strongly oxidizing conditions.

\section{Discussion}

The Füzes basalts originated by Hawaiian to Strombolian explosive volcanic eruptions and formed a scoria cone. Based on the available age data (Balogh et al. 1982, 1986; Borsy et al. 1986; Balogh and Pécskay 2001; Wijbrans et al. 2007), this was probably the last volcanic eruption in the Bakony-Balaton Highland Volcanic Field. The volcanic bombs and the relatively massive blocks show fairly uniform petrographic character, namely they contain abundant xenocrysts. Alkaline basalt with similar features is known in the Bondoró area. Due to the common ultramafic xenolith fragments and the abundant xenocrysts the volcanic products do not represent the original composition of the basaltic melt formed in the upper mantle. This is reflected in their bulk major and trace element contents having extremely high $\mathrm{MgO}$ content and $\mathrm{Ni}$ and $\mathrm{Cr}$ concentrations. This geochemical composition also resembles the Bondoró basalt (Embey-Isztin et al. 1993). The enrichment in the highly incompatible trace elements and the relative depletion in the heavy rare earth elements (Fig. 5) imply a low degree of partial melting. The slight negative potassium anomaly could be attributed to the 
Table 6

Representative compositions of the studied spinels. $\mathrm{Fe}_{2} \mathrm{O}_{3}$ is calculated on the basis of stoichiometry

\begin{tabular}{|c|c|c|c|c|c|c|}
\hline & \multicolumn{2}{|c|}{$\begin{array}{c}\text { brownish green } \\
\text { xenocryst }\end{array}$} & \multicolumn{2}{|c|}{ dark brown xenocryst } & \multicolumn{2}{|c|}{ light brown xenocryst } \\
\hline & sp1 & sp2 & sp3 & sp4 & sp5 & sp6 \\
\hline $\mathrm{SiO}_{2}$ & 0.03 & 0.03 & 0.04 & 0.04 & 0.07 & 0.04 \\
\hline $\mathrm{TiO}_{2}$ & 0.09 & 0.12 & 0.12 & 0.08 & 0.23 & 0.25 \\
\hline $\mathrm{Al}_{2} \mathrm{O}_{3}$ & 57.65 & 54.17 & 36.23 & 29.15 & 47.41 & 43.92 \\
\hline $\mathrm{Cr}_{2} \mathrm{O}_{3}$ & 9.39 & 12.20 & 30.38 & 36.20 & 20.42 & 22.60 \\
\hline $\mathrm{Fe}_{2} \mathrm{O}_{3}$ & 2.06 & 2.57 & 4.03 & 5.88 & 2.47 & 3.41 \\
\hline $\mathrm{FeO}$ & 10.15 & 10.21 & 11.51 & 12.32 & 9.32 & 11.51 \\
\hline $\mathrm{MnO}$ & 0.10 & 0.09 & 0.15 & 0.18 & 0.07 & 0.12 \\
\hline $\mathrm{NiO}$ & 0.41 & 0.38 & 0.26 & 0.22 & 0.34 & 0.27 \\
\hline $\mathrm{MgO}$ & 19.96 & 19.48 & 16.89 & 15.61 & 19.66 & 17.86 \\
\hline Total & 99.84 & 99.23 & 99.61 & 99.66 & 99.99 & 99.98 \\
\hline $\mathrm{Mg} \#\left(\mathrm{Fe}^{\mathrm{tot}}\right)$ & 0.75 & 0.73 & 0.67 & 0.61 & 0.75 & 0.69 \\
\hline \multirow[t]{3}{*}{$\mathrm{Cr} \#$} & 9.85 & 13.12 & 36.00 & 45.45 & 22.42 & 25.66 \\
\hline & \multicolumn{4}{|c|}{ inclusion in ol phenocryst } & \multicolumn{2}{|c|}{ in opx reaction rim } \\
\hline & $\mathrm{sp} 7$ & $\mathrm{sp} 8$ & sp9 & sp10 & sp11 & sp12 \\
\hline $\mathrm{SiO}_{2}$ & 0.17 & 0.14 & 0.11 & 0.11 & 0.21 & 1.35 \\
\hline $\mathrm{TiO}_{2}$ & 0.88 & 0.88 & 0.64 & 1.11 & 2.87 & 3.52 \\
\hline $\mathrm{Al}_{2} \mathrm{O}_{3}$ & 40.86 & 36.19 & 37.13 & 35.90 & 5.63 & 2.75 \\
\hline $\mathrm{Cr}_{2} \mathrm{O}_{3}$ & 20.40 & 23.02 & 21.50 & 21.87 & 17.68 & 23.00 \\
\hline $\mathrm{Fe}_{2} \mathrm{O}_{3}$ & 5.52 & 7.15 & 7.71 & 7.80 & 35.91 & 35.24 \\
\hline $\mathrm{FeO}$ & 15.54 & 17.37 & 17.47 & 18.59 & 25.76 & 12.04 \\
\hline $\mathrm{MnO}$ & 0.18 & 0.22 & 0.36 & 0.28 & 0.54 & 1.51 \\
\hline $\mathrm{NiO}$ & 0.21 & 0.19 & 0.17 & 0.29 & 0.24 & 0.25 \\
\hline $\mathrm{MgO}$ & 15.14 & 13.32 & 13.02 & 12.47 & 4.27 & 13.69 \\
\hline Total & 98.90 & 98.48 & 98.12 & 98.42 & 93.11 & 93.34 \\
\hline $\mathrm{Mg} \#\left(\mathrm{Fe}^{\mathrm{tot}}\right)$ & 0.57 & 0.50 & 0.49 & 0.46 & 0.12 & 0.36 \\
\hline $\mathrm{Cr} \#$ & 25.09 & 29.91 & 27.98 & 29.01 & 67.80 & 84.87 \\
\hline
\end{tabular}

$\mathrm{sp}=$ spinel; ol=olivine; opx= orthopyroxene; $\mathrm{Mg} \#\left(\mathrm{Fe}_{\mathrm{tot}}\right)=\mathrm{Mg} /\left(\mathrm{Mg}+\mathrm{Fe}_{\mathrm{tot}}\right) ; \mathrm{Cr} \#=100^{*} \mathrm{Cr} /(\mathrm{Cr}+\mathrm{Al})$ 
presence of a K-bearing phase in the source region, either amphibole or phlogopite. However, during the volcanic eruption, potassium was preferentially depleted, possibly during the degassing process as indicated by the more significant K-depletion in the scoria. Ratios of the highly incompatible trace elements, which do not enter the olivine, pyroxene and spinel xenocrysts can be used to infer on the origin of the primary magma. $\mathrm{Zr} / \mathrm{Nb}$ and $\mathrm{Nb} / \mathrm{Y}$ ratios of the studied samples (Fig. 8) indicate less than 1\% of partial melting in the transitional spinel-garnet stability field according to Harangi (2002). This sets the melt generation process at $70-80 \mathrm{~km}$ depth, i.e. the uppermost part of the asthenosphere (as for the other alkaline basalts in the region; Embey-Isztin et al. 1993; Harangi et al. 1994).

The studied basaltic rocks are rich in crystals, which provide further information on the source region of the magma, about the nature of the sublithospheric mantle, as well as on the early crystallization processes. In the

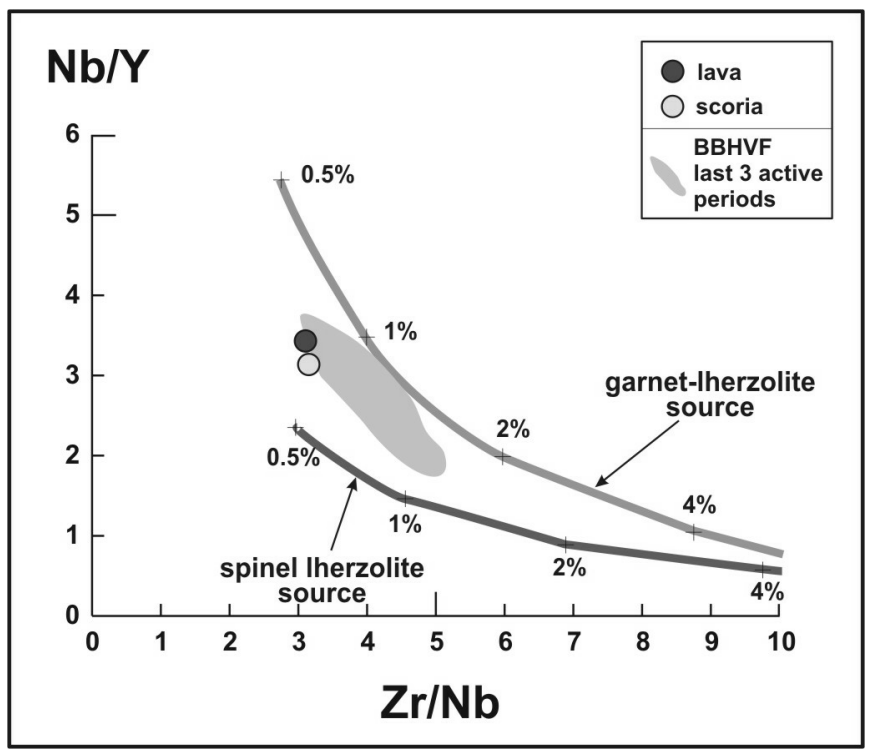

Fig. 8

$\mathrm{Zr} / \mathrm{Nb}$ vs. $\mathrm{Nb} / \mathrm{Y}$ diagram after Harangi (2002). The numbers with percentages indicate the degree of partial melting. Alkaline rocks of the Balaton Highland (Embey-Isztin et al. 1993; Harangi et al. 1994) are also plotted which represent the last three active periods of the volcanic activity

following we discuss the messages hidden in the chemical composition of the mineral constituents of the Füzes basalt.

\section{Implications on the nature of the source region}

Compositions of magmatic spinel inclusions in olivine phenocrysts provide important information about the melt composition (Roeder 1994) and its source region (Arai 1992, 1994). In addition, spinel composition is also affected by the $\mathrm{fO}_{2}$ (e.g. Roeder and Reynolds 1991), crystallization temperature (e.g. Thy 1995), 
pressure (e.g. Sigurdsson and Schilling 1976), melt differentiation (e.g. Arai 1994) and/or magma mixing/mingling (e.g. Natland et al. 1983; Kamenetsky and Crawford 1998). "However, when carefully screened to minimize these effects, compositions of spinel inclusions and host olivine phenocrysts have proved to be useful indicators of the most primitive aspects of melt composition (Clynne and Borg 1997), and thus the nature of partial melting processes and mantle sources (e.g. Dick and Bullen 1984)" (Smith and Leeman 2005). Spinel is the earliest, often liquidus phase in the crystallization history of mantle-derived basaltic melts and its composition is similar to the spinel in the residual mantle source (Arai 1994).

Olivine phenocrysts of the Füzes basalt often contain spinel inclusions. Figure 9a illustrates the relationship between the Fo content of the host olivine and the Cr-number of co-precipitated spinel. The sample points form a roughly horizontal trend at relatively low spinel Cr-number (around 30), which is between the dark brown and light brown xenocrystic, mantle-derived spinel populations. By extrapolating this trend back to the olivine-spinel mantle array (OSMA; Arai 1992, 1994), the nature of the residual mantle source could be inferred assuming that the primary Mg-rich magma was in chemical equilibrium with its mantle restite. Using this concept, the Füzes basalt could have derived from a slightly depleted peridotite source. The horizontal trend (Fig. 9a) suggests that only olivine was crystallized along with the spinel during the early stage of the basaltic magma evolution, since the $\mathrm{Cr} \#$ of spinel is almost constant with decreasing Fo of olivine. This implies that no other minerals, which incorporate either $\mathrm{Al}$ (e.g. plagioclase) or $\mathrm{Cr}$ (e.g. clinopyroxene), crystallized along with the spinels. Based on the low Cr-number of the spinels the mantle source of the Füzes basalt differs significantly from other localities (Pauliberg, Uzsa, Ság Hill, and

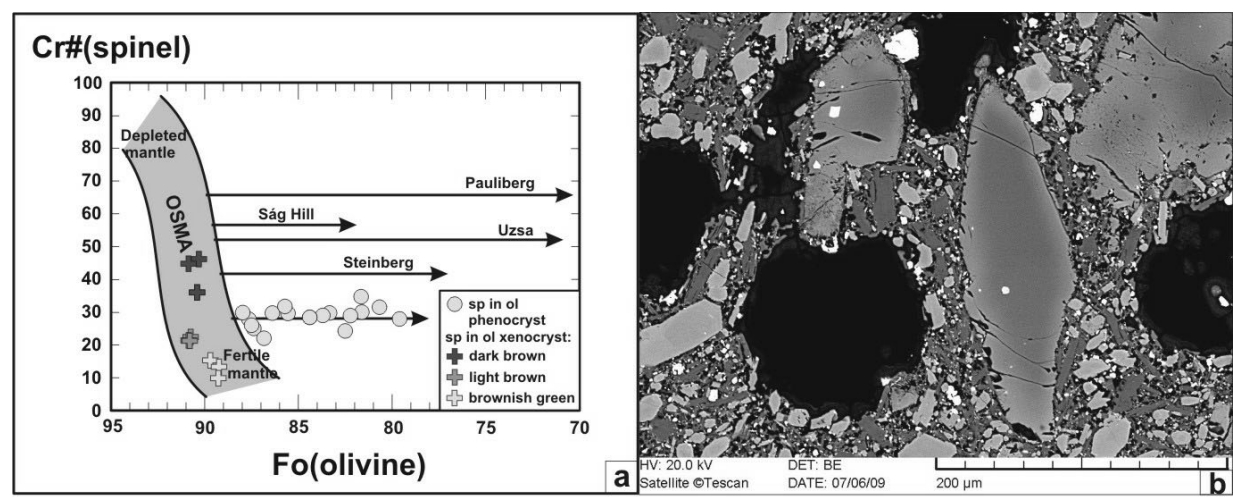

Fig. 9

a) Fo content of host olivine vs. Cr-number of their spinel inclusions for the studied pheno- and xenocrystic olivine-spinel pairs (Arai 1994). OSMA - olivine-spinel mantle array. The trend of the phenocrystic olivine-spinel pairs of the other alkaline basalts from the western Pannonian Basin (Pauliberg, Uzsa, Ság Hill and Steinberg: Sági 2008) are also plotted; b) Subhedral olivine phenocrysts with Cr-spinel inclusions (BSE image) 
Steinberg; Sági 2008) in the western Pannonian Basin (Fig. 9a). Compared with the others, the olivine-spinel pairs of the Füzes-tó basalt implies the least depleted residual mantle source according to Arai's method (Arai 1994).

The general view is that the $\mathrm{Cr}$-number $(\mathrm{Cr} /(\mathrm{Cr}+\mathrm{Al}))$ of spinel in primitive basalts is a reflection of the extent of depletion of the mantle peridotite source (e.g. Roeder 1994). The low Cr\# of spinel reflects a fertile peridotite source that has not seen a previous generation of melting, thus there is considerable $\mathrm{Al}$ in the peridotite for the melt. Based on this the low-Cr\# spinel inclusions of the Füzestó basalt suggest a fertile mantle peridotite source.

Kamenetsky et al. (2001) introduced an $\mathrm{Al}_{2} \mathrm{O}_{3}-\mathrm{TiO}_{2}$ diagram to discriminate between spinels that crystallized from different magmas in different geodynamic settings. In addition, this diagram can be useful to determine the tectonic setting of spinels from altered mafic igneous rocks and detrital spinels in sandstones. The $\mathrm{Al}_{2} \mathrm{O}_{3}$ and $\mathrm{TiO}_{2}$ content of volcanic $\mathrm{Cr}$-spinel depend on the parental melt composition, which is a function of pressure, temperature, degree of partial melting, and source chemical and phase composition (Kamenetsky et al. 2001). On the $\mathrm{Al}_{2} \mathrm{O}_{3}$ vs. $\mathrm{TiO}_{2}$ diagram (Fig. 10) spinels of the alkaline basalts in the western Pannonian Basin forms a noticeable negative trend with the Füzes basalt at the low-Ti, high-Al end. This trend is coincident with the changes in the petrological-geochemical characteristics of the mantle and conditions of mantle melting (Kamenetsky et al. 2001). Since the melting conditions are very similar in the case of the alkaline basalts in the western Pannonian Basin, a small-scale heterogeneity can be assumed in the upper mantle. Most of the spinels from the Füzes basalt plots into the MORB field, whereas others are around the OIB field. The spinels from Pauliberg has characteristically high $\mathrm{TiO}_{2}$ concentration reflecting the Ti-rich composition of the host basalt (Sági 2008) and thus they form a separate group. The spinel compositions of the Füzes basalt resemble the MORB-spinels, but it is obvious that the studied basalt does not derive from the

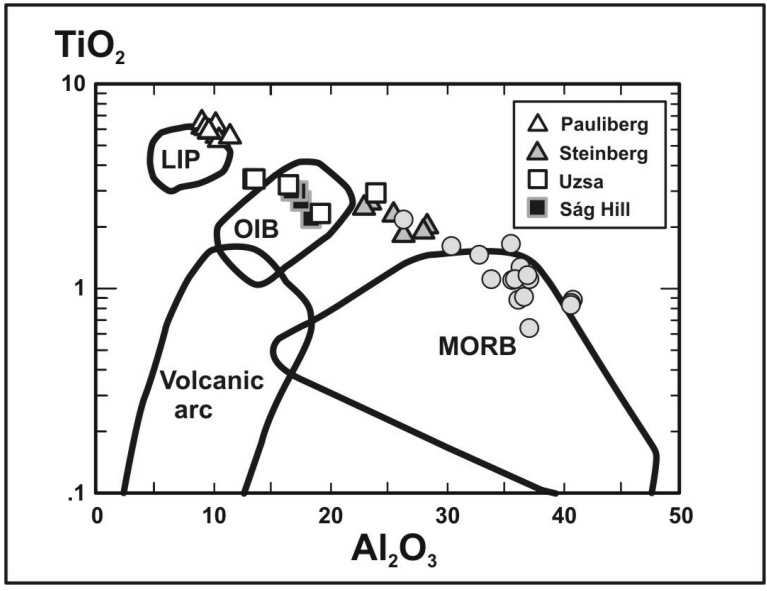

Fig. 10

$\mathrm{Al}_{2} \mathrm{O}_{3}$ vs. $\mathrm{TiO}_{2}$ compositional relationship in the studied spinel inclusions (grey circles) of olivine phenocrysts (Kamenetsky et al 2001). LIP - Large Igneous Provinces, OIB - Ocean Island Basalts, MORB - Mid-Ocean Ridge Basalts. Spinel compositions of the other alkaline basalts (Pauliberg, Uzsa, Ság Hill and Steinberg: Sági 2008) are also plotted 
geodynamic setting of a mid-oceanic ridge. The similar (i.e. Al-rich) spinel compositions in the Füzes basalt can reflect fertile mantle source.

\section{The origin of the xenocrysts}

The Füzes basalt contains abundant xenocrysts such as olivine, clino- and orthopyroxene and spinel. They could be derived from the subcontinental lithospheric mantle or represent high-pressure cumulate phases. The compositions of the olivine, orthopyroxene, colourless clinopyroxene and spinel resemble those of the main mineral phases of the ultramafic xenoliths enclosed by the basalts in the Bakony-Balaton Highland Volcanic Field (Embey-Isztin et al. 2001a); thus most of them could be fragments incorporated from the lithospheric mantle.

Mantle mineral compositions are sensitive to the degree of depletion of their source mantle peridotite. The $\mathrm{Al}, \mathrm{Ti}, \mathrm{Ca}$ and $\mathrm{Na}$ enter preferentially into the basaltic melt during partial melting, so the residual minerals in the peridotite will be depleted in these elements. On the other hand, $\mathrm{Mg}, \mathrm{Fe}, \mathrm{Cr}$ and $\mathrm{Ni}$ contents of the peridotite and their mineral phases increase, since these elements are particularly enriched in the residual phases during partial melting. In the following, compositions of the xenocrysts are compared with a large database of the mineral phases from ultramafic xenoliths of Bondoró (Embey-Isztin et al. 2001a; unpublished data, Matalin 2008).

The Füzes olivine xenocrysts are Fo-rich $\left(\mathrm{Fo}_{89-91}\right)$, low-Ca $(<0.1 \mathrm{wt} \%)$ and high$\mathrm{Ni}(\mathrm{NiO}=0.36-0.42 \mathrm{wt} \%)$ crystals, which are more primitive than the olivine phenocrysts (Table 3) (see also in Embey-Isztin and Dobosi 2007). Olivine with such composition is generally interpreted as a mantle xenocryst (e.g. Ramsay et al. 1984; Boudier 1991; Hirano et al. 2004; Rohrbach et al. 2005), which were picked up accidentally from the mantle through which the magma ascended.

Compositions of the colourless clinopyroxene xenocrysts, found as variably resorbed cores in phenocrysts, indicate mainly moderately a depleted peridotite source based on their similarity to the mantle clinopyroxenes of Bondoró (lherzolite-harzburgite transition; Fig. 11a), and only one of them resembles the diopsides of fertile lherzolite. The orthopyroxene compositions support this interpretation, since most of them have $\mathrm{Al}_{2} \mathrm{O}_{3}$ and $\mathrm{MgO}$ content similar to those found in variably depleted peridotites (Fig. 11b). In addition, one sample could represent a refractory, whereas another one reflects a fertile mantle lithology.

In contrast the spinel xenocrysts indicate a much wider compositional range (Fig. 11c), as suggested also by their various colour. Their $\mathrm{Al}_{2} \mathrm{O}_{3}$ content varies between 27 and $59 \mathrm{wt} \%$, whereas their Cr-number is in the range between 10 and 48. Remarkably, this compositional range roughly overlaps the data of spinels from the Bondoró ultramafic xenoliths. The basaltic magma could have sampled the entire lithospheric mantle at various depths, and picked up smaller and larger fragments from peridotites with various lithologies. This lithological and geo- 
chemical variation could be similar to what is shown by the ultramafic xenoliths from Bondoró. It might be assumed that the pyroxenes underwent more intense corrosion during the transport in the basaltic melt, whereas spinel was more resistant and thus better preserved the variability of the lithospheric mantle.

A characteristic feature of the orthopyroxene xenocrysts in the Füzes basalt is that they are usually surrounded by a reaction rim, which is a characteristic product of reaction between orthopyroxene and melt (Arai and Abe 1995; Shaw et al. 1998). In some cases it is composed of two subzones: a fine-grained inner

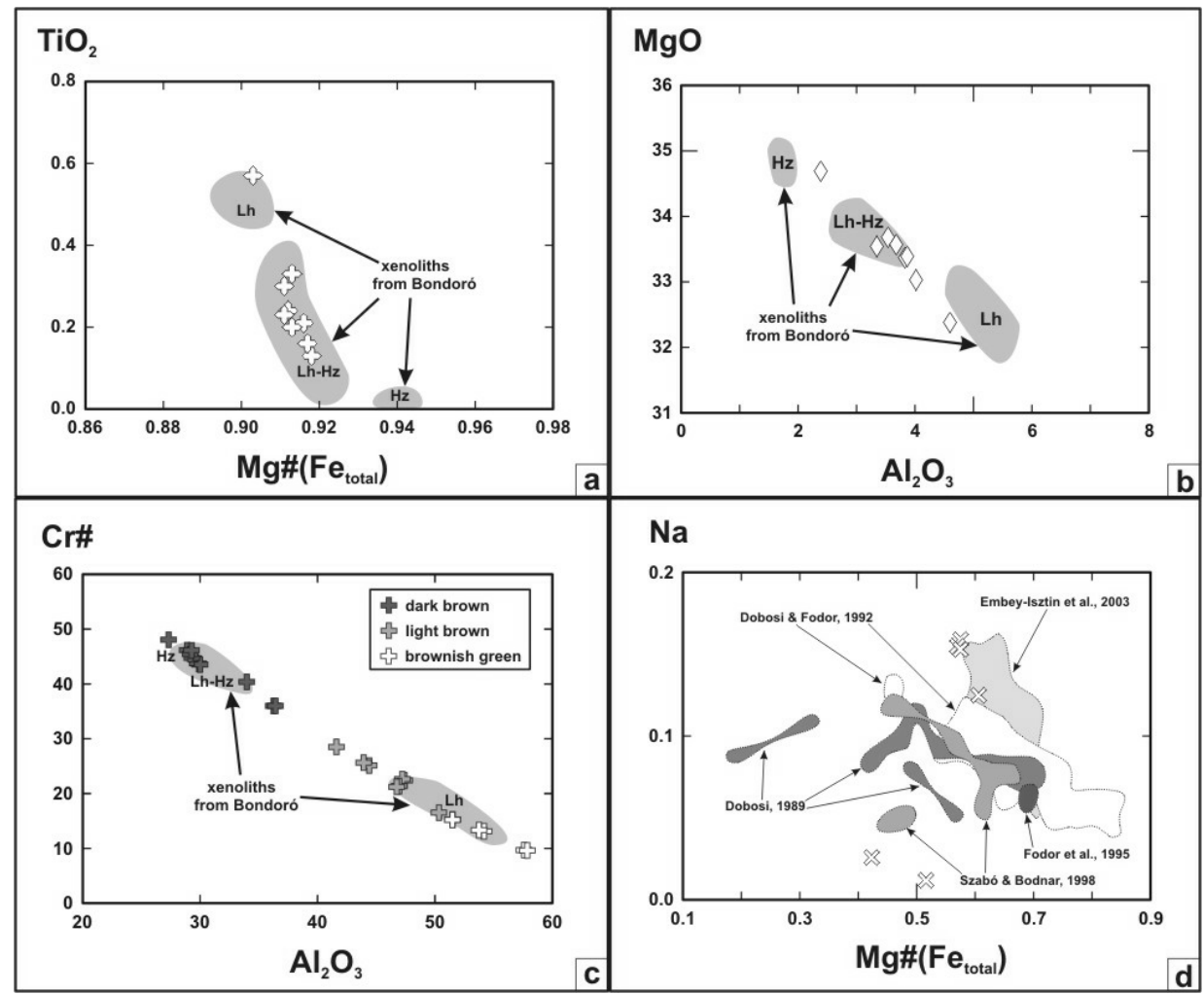

Fig. 11

a) Mg-number $\left(\mathrm{Fe}_{\text {total }}\right)$ vs. $\mathrm{TiO}_{2}$ content in the studied colourless clinopyroxene xenocrysts. Clinopyroxene compositions of xenoliths from Bondoró Hill (Embey-Isztin et al. 2001a; unpublished data, Matalin 2008) are plotted with grey fields. $\mathrm{Lh}$ - lherzolite; $\mathrm{Lh}-\mathrm{Hz}$ - lherzolite-harzburgite; $\mathrm{Hz}-$ harzburgite; b) Relationship between $\mathrm{Al}_{2} \mathrm{O}_{3}$ and $\mathrm{MgO}$ content of the studied orthopyroxene xenocrysts. Orthopyroxene compositions of xenoliths from Bondoró Hill (Embey-Isztin et al. 2001a; unpublished data, Matalin 2008) are plotted with grey fields; c) $\mathrm{Al}_{2} \mathrm{O}_{3}$ content vs. $\mathrm{Cr}$ - number in the studied spinel xenocrysts. $\mathrm{Cr} \#-100^{*} \mathrm{Cr} /(\mathrm{Cr}+\mathrm{Al})$. Spinel compositions of xenoliths from Bondoró Hill (Embey-Isztin et al. 2001a; unpublished data, Matalin 2008) are plotted with grey fields, d) Relationship between $\mathrm{Mg}$-number $\left(\mathrm{Fe}_{\text {total }}\right)$ vs. $\mathrm{Na}$ content in the studied green clinopyroxene xenocrysts. The compositions of other green clinopyroxenes (Dobosi 1989; Dobosi and Fodor 1992; Fodor et al. 1995; Szabó and Bodnar 1998; Embey-Isztin et al. 2003) are plotted with dashed line fields 
(adjacent to orthopyroxene) and a coarser-grained outer one (adjacent to the host basalt; Fig. 4b). These two subzones consist basically of olivine + clinopyroxene + glass \pm spinel. Spinel was observed only in the outer subzone. The interaction between the alkaline basalt and orthopyroxene is a dynamic disequilibrium process, which possibly depends on the composition of the orthopyroxene, the rate of supply of undersaturated melt and other processes (Daines and Kohlstedt 1994). The reaction zone can also be interpreted as a melt-mixing zone, where the Si-rich secondary melt, derived from the incongruent melting of orthopyroxene, is mixed with the surrounding, less silicic alkaline basaltic melt (Arai and Abe 1995). In the system $\mathrm{Fo}+\mathrm{SiO}_{2}$ enstatite breaks down at $1559{ }^{\circ} \mathrm{C}$ and at 1 atmosphere to form forsterite and silica, and the amount of olivine that should be formed on incongruent melting is approximately 5-6\% (Bowen and Anderson 1914). We observed approximately $40-50 \%$ olivine in the reaction rim. The additional olivine could crystallize from the melt of the hybrid boundary layer, which was formed by the mixing of the basanite melt and the liquid derived from orthopyroxene breakdown (Shaw et al. 1998). The proportion of clinopyroxene is approximately $30-40 \%$ in the studied reaction rims. Clinopyroxene is not a normal product of the incongruent breakdown of orthopyroxene; the components required for its formation must have diffused into the boundary layer from the basanite melt (Shaw et al. 1998). Glass occurs in amounts of approximately $10-20 \%$ in the studied reaction zones. Accordingly, the studied reaction rim of the orthopyroxene xenocrysts could be the product of the interaction between the alkaline basaltic melt and the mantle-derived orthopyroxene. This reaction could have occurred at pressures lower than approximately $5 \mathrm{kbar}$, since according to Shaw et al. (1998) at pressures greater than about $5 \mathrm{kbar}$ the breakdown of orthopyroxene is no longer incongruent.

The sporadic green, Fe-rich clinopyroxenes in the cores of some clinopyroxene phenocrysts have a distinct composition from the others. they have low Ti/Al ratio and typically low Mg-number $(<0.6)$. Thus they might be derived from high-pressure crystallization of a more evolved melt compared with the host basalt. The origin of such green clinopyroxenes has been studied extensively during the past decades and this has resulted in different interpretations (e.g. Brooks and Printzlau 1978; Wass 1979; Duda and Schmincke 1985). Dobosi (1989) described two kinds of anhedral green clinopyroxene cores in the Nógrád basalts: olive-green (fassaites and fassaitic augites) and grass-green (salites and ferrosalites). The olive-green pyroxenes are considered to have been crystallized from a more evolved melt which subsequently mixed with the mafic host magma (e.g. Brooks and Printzlau 1978), whereas the salitic and ferrosalitic green clinopyroxenes could have been derived from disaggregated xenoliths of metasomatized upper mantle (Barton and Bergen 1981). Dobosi and Fodor (1992) studied green-core clinopyroxenes in the basalts of the Nógrád-Fil'akovo province. They also stated that the fassaitic green cores were formed by fractionation, replenishment and convective mixing within the magma reservoir 
of the host lavas. The Fe-rich diopside cores were interpreted as wall-rock xenocrysts. Fodor et al. (1995) studied Fe-rich diopsides occurring as anhedral, resorbed cores within cognate clinopyroxene (in northeastern Brazil), and they supported their xenocrystic origin as well. Szabó and Bodnar (1998) distinguished two groups of green clinopyroxenes in the alkaline basalts of the Nógrád-Gömör Volcanic Field. Group 1 clinopyroxenes (Al-rich), which also occurs in clinopyroxenite xenoliths, were interpreted as xenocrysts from the uppermost mantle. The Group 2 clinopyroxenes (Al-poor) could probably represent xenocrysts from disaggregated dioritic cumulates produced from melts related to the host basanitic magmas. In addition, green clinopyroxenes could, however, also be a major constituents of lower crustal mafic granulite xenoliths which have been found in the Bakony-Balaton Highland Volcanic Field (EmbeyIsztin et al. 1990, 2003) and in the Nógrád-Gömör Volcanic Field (Kovács and Szabó 2005).

The green clinopyroxenes of the Füzes-tó basalt can be divided into two groups (Fig. 11d): there is a Na-rich group with a composition similar to those of the clinopyroxenes found in lower crustal granulite xenoliths from the Balaton Highland area (Embey-Isztin et al. 2003). A second, Na-poor group, differs from all the plotted green clinopyroxenes in Fig. 11d, so they probably have a different origin. For example, green clinopyroxenes are common in different crustal rocks representing the middle and lower levels (personal comm., Török 2009); thus further investigations are needed to determine their origin.

\section{Crystallization of the Füzes-tó basalt}

In addition to the vast amount of xenocrysts, the Füzes basalt contains various phenocrysts such as olivine and clinopyroxene. The latter is not so common as phenocrysts in the basalts of the Bakony-Balaton Highland Volcanic Field (Embey- Isztin et al. 1993). In the olivine phenocrysts the Ca and Mn show correlations with the Fo contents (Figs 6 and 12). The Ca content of olivine is a function of pressure, host magma chemical composition and Fo content of olivine (Simkin and Smith 1970; Stormer 1973; Libourel 1999). The increasing Ca content of the phenocrysts observed from their cores to the rims (Fig. 6) implies that their crystallization occurred during continuously decreasing pressure (e.g. Stormer 1973). The thin rims around the olivine xenocrysts were formed during this polybaric crystallization. The $\mathrm{Mn}$ and Fe show a positive correlation in the olivines as a rough linear trend (Fig. 12). The slope of this trend is larger than that characterized by olivine from other localities (Ság and Pauliberg; Sági 2008). The steeper Fe-Mn trend suggests lower a Fe/Mn ratio. This feature could be explained by difference in the redox condition of the crystallization and imply a strongly oxidized environment for the Füzes-tó basaltic magma. This is consistent with the characteristic reddish colour of the basaltic bombs, the iddingsitization of the olivine phenocrysts and with the strongly altered peridotite xenoliths. The 
Fig. 12

Fe vs. Mn (atomic values) compositional relationship in the studied olivines. The thick grey line indicates the Fe-Mn trend of the studied olivines, and the Fe-Mn trends of the olivines from Pauliberg and Ság Hill (Sági 2008) are also plotted

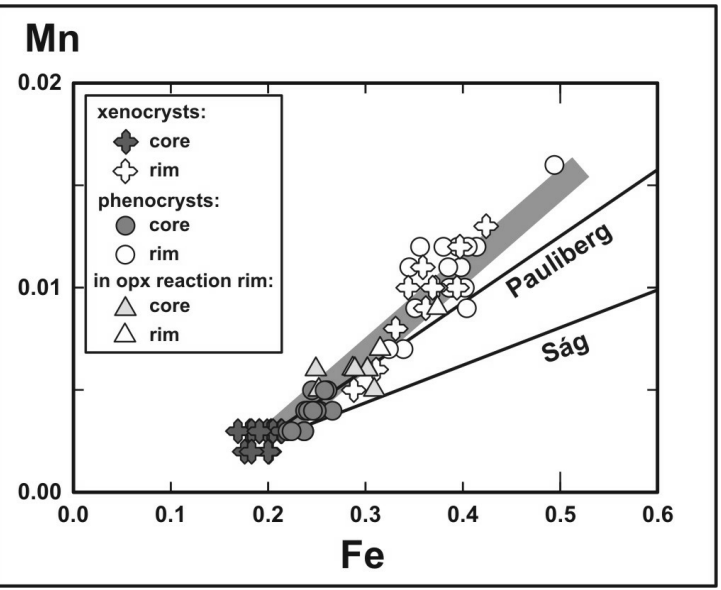

slight change in the $\mathrm{Fe}-\mathrm{Mn}$ trend at $\mathrm{Fe}=0.33$ a.p.f.u. suggests, however, that this oxidizing condition prevailed mostly at the later stages of the crystallization.

Compositions of the clinopyroxenes are also dependant on the circumstances of the crystallization history. The $\mathrm{Ti}$ and $\mathrm{Al}$ content and the $\mathrm{Ti} / \mathrm{Al}$ ratio of the clinopyroxenes could provide qualitative information about the pressure of their crystallization, since more Ti can be incorporated in the clinopyroxene lattice with decreasing pressure (Yagi and Onuma 1967; Dobosi et al. 1991). Both the pheno- and microphenocrysts enrich in $\mathrm{Al}$ and $\mathrm{Ti}$ from zone1 to zone2 in the zoned clinopyroxenes (Fig. 7a). Their Ti/Al ratio shows a slight increase from zone1 to zone2 indicating crystallization under continuously decreasing pressure. This clinopyroxene evolution was accompanied with a strong enrichment in Ti with decreasing Mg-number (Fig. 7b), which reflects the normal fractionation trend and has been found at several alkaline basalt suites (Tracy and Robinson 1977; Bédard et al. 1988).

\section{Conclusions}

The mineral-scale investigation of the Füzes-tó basalt has revealed the main processes occurring from partial melting process up to the eruption (Fig. 13). The main phases of the petrogenetic evolution are summarized below (see the numbers in Fig. 13):

I. The primary magma could have originated at the uppermost part of the asthenosphere by very low-degree partial melting (probably less than $1 \%$; Fig. 8) in the transitional spinel-garnet lherzolite stability field, i.e. about at 70-80 km depth. The relatively low $\mathrm{Cr} \#$ of spinel inclusions in olivine phenocrysts can reflect fertile mantle peridotite source (e.g. Roeder 1994).

II. The basaltic magma ascended rapidly toward the surface, while it picked up smaller and larger fragments as xenocrysts and xenoliths from different regions of the subcontinental lithospheric mantle. 


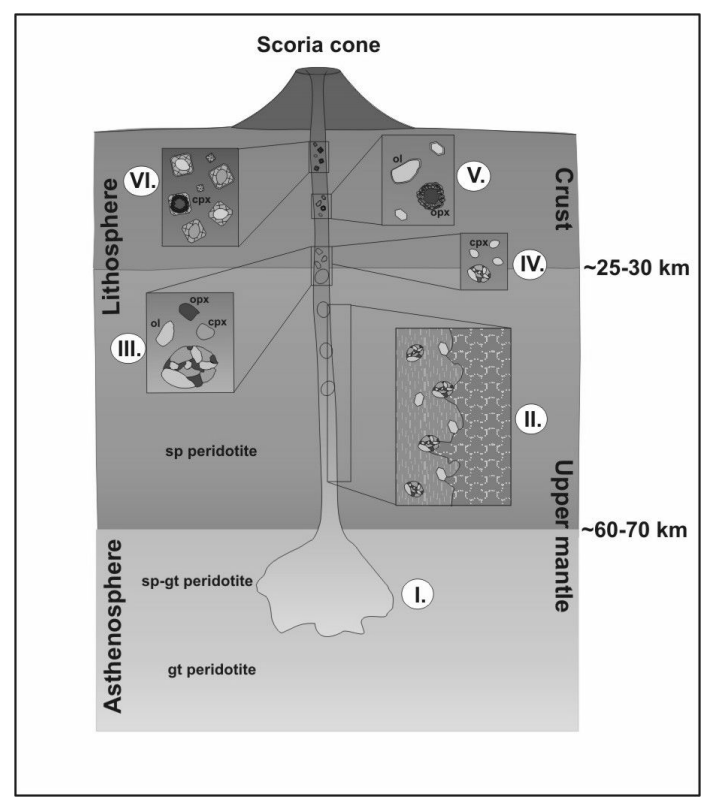

Fig. 13

Petrogenetic model for the alkaline basaltic magma of the Füzes-tó scoria cone. The numbers indicate the main phases of the magma evolution (gt garnet, sp - spinel, ol - olivine, cpx clinopyroxene, opx - orthopyroxene)

III. These fragments and minerals underwent strong resorbtion in the magma and most of the xenoliths disaggregated.

IV. In the lower crustal levels Fe-rich green clinopyroxenes (at least some of the studied green clinopyroxenes) were incorporated into the ascending mafic magma.

V. Near-liquidus olivines (based on their high Fo content) crystallized from the alkaline basaltic magma along with Al-rich spinels. This was followed by the dissolution of orthopyroxene xenocrysts and the formation of a reaction rim typical of melt-orthopyroxene interaction. The incongruent dissolution of the orthopyroxene occurs only at pressures lower than approximately $5 \mathrm{kbar}$ (Shaw et al. 1998) corresponding to approximately $18 \mathrm{~km}$ depth. Therefore this reaction rim (composed of olivine + clinopyroxene $+\mathrm{Si}$ rich glass \pm spinel) could have been formed at depths shallower than $15 \mathrm{~km}$. Thereafter, a magnesian-poor rim crystallized both on the olivine xeno- and phenocrysts from the more evolved magma.

VI. The resorbed clinopyroxene xenocrysts and the reaction rim of the orthopyroxene xenocrysts could act as nucleation sites for the subsequent clinopyroxene crystallization. Zoned clinopyroxene pheno- and microphenocrysts were formed during continuously decreasing pressure.

\section{Acknowledgements}

We gratefully thank Dr. Gábor Dobosi and Professor Ron Fodor for their useful suggestions and comments in their reviews that considerably improved the manuscript. We are very thankful for the subsidy of the Pro Renovanda Cultura Hungariae Student Science Foundation. We also thank Balázs Kiss and Tamás Sági for their help with the sample collection and analysis, Zsolt Bendo for use of the SEM, Csaba Szabó for the useful information about the Füzes-tó area and Kálmán Török for the discussions about the origin of the green clinopyroxenes. 


\section{References}

Aoki, K., I. Kushiro 1968: Some clinopyroxenes from ultramafic inclusions in Dreiser Weiher, Eifel. Contributions to Mineralogy and Petrology, 18, pp. 326-337.

Arai, S. 1992: Chemistry of chromian spinel in volcanic rocks as a potential guide to magma chemistry. - Mineralogical Magazine, 56, pp. 173-184.

Arai, S. 1994: Compositional variation of olivine-chromian spinel in Mg-rich magmas as a guide to their residual spinel peridotites. - Journal of Volcanology and Geothermal Research, 59, pp. 279-293.

Arai, S., N. Abe 1995: Reaction of orthopyroxene in peridotite xenoliths with alkali-basalt melt and its implication for genesis of alpine-type chromitite. - American Mineralogist, 80, pp. 1041-1047.

Auer, A., U. Martin, K. Németh 2007: The Fekete-hegy (Balaton Highland Hungary) "soft-substrate" and "hard-substrate" maar volcanoes in an aligned volcanic complex - Implications for vent geometry, subsurface stratigraphy and the palaeoenvironmental setting. - Journal of Volcanology and Geothermal Research, 159, pp. 225-245.

Balogh, K., A. Jámbor, Z. Partényi, L. Ravaszné Baranyai, G. Solti 1982: K/Ar radiogenic age of Transdanubian basalts (in Hungarian with English summary). - MÁFI Évi Jel. 1980-ről, pp. 243-259.

Balogh, K., E. Árva-Sós, Z. Pécskay, L. Ravasz-Baranyai 1986: K/Ar dating of post Sarmatian alkali basaltic rocks in Hungary. - Acta Mineralogica et Petrographica Szeged, 28, pp. 75-94.

Balogh, K., Z. Pécskay 2001: K/Ar and Ar/Ar geochronological studies in the Pannonian-Carpathians-Dinarides (PANCARDI) region. - Acta Geol. Hung., 44, pp. 281-301.

Barton, M., v.M.J. Bergen 1981: Green clinopyroxenes and associated phases in a potassium-rich lava from the Leucite Hills, Wyoming. - Contributions to Mineralogy and Petrology, 77, pp. 101-114.

Bédard, J.H.J., D.M. Francis, J. Ludden 1988: Petrology and pyroxene chemistry of Monteregian dykes: the origin of concentric zoning and green cores in clinopyroxenes from alkali basalts and lamprophyres. - Canadian Journal of Earth Sciences, 25, pp. 2041-2058.

Borsy, Z., K. Balogh, M. Kozák, Z. Pécskay 1986: Contributions to the evolution of the Tapolca-basin, Hungary (in Hungarian with English abstract). - Acta Geographica Debrecina, 23, pp. 79-104.

Boudier, F. 1991: Olivine xenocrysts in picritic magmas: An experimental and microstructural study. - Contributions to Mineralogy and Petrology, 109, pp. 114-123.

Bowen, N.L., O. Anderson 1914: The binary system $\mathrm{MgO}-\mathrm{SiO}_{2}$ - - American Journal of Science, 37, pp. 487-500.

Brooks, C.K., I. Printzlau 1978: Magma mixing in mafic alkaline volcanic rocks: The evidence from relict phenocryst phases and other inclusions. - Journal of Volcanology and Geothermal Research, 4, pp.

Clynne, M.A., L.E. Borg 1997: Olivine and chromian spinel in primitive calc-alkaline and tholeiitic lavas from the southernmost Cascade Range, California; a reflection of relative fertility of the source. - Canadian Mineralogist, 35, pp. 453-472.

Csillag, G. 2004: Geomorphologic levels of the Kál Basin and its vicinity (in Hungarian with English abstract). - MÁFI Évi Jel. 2002-ról, pp. 95-110.

Daines, M.J., D.L. Kohlstedt 1994: The Transition from Porous to Channelized Flow Due to Melt/Rock Reaction During Melt Migration. - Geophysical Research Letters, 21, pp. 145-148.

Dick, H.J.B., T. Bullen 1984: Chromian spinel as a petrogenetic indicator in abyssal and alpine-type peridotites and spatially associated lavas. - Contributions to Mineralogy and Petrology, 86, pp. $54-76$.

Dobosi, G. 1989: Clinopyroxene zoning patterns in the young alkali basalts of Hungary and their petrogenetic significance. - Contributions to Mineralogy and Petrology, 101, pp. 112-121.

Dobosi, G., R. Schultz-Güttler, G. Kurat, A. Kracher 1991: Pyroxene chemistry and evolution of alkali basaltic rocks from Burgenland and Styria, Austria. - Mineralogy and Petrology, 43, pp. 275-292. 
Dobosi, G., R.V. Fodor 1992: Magma fractionation, replenishment, and mixing as inferred from green-core clinopyroxenes in Pliocene basanite, southern Slovakia. - Lithos, 28, pp. 133-150.

Dobosi, G., R.V. Fodor, S.A. Goldberg 1995: Late-Cenozoic alkali basalt magmatism in Northern Hungary and Slovakia: Petrology, source compositions and relationship to tectonics. - In: H. Downes, O. Vaselli (Eds): Neogene and Related Magmatism in the Carpatho-Pannonian Region. Acta Vulcanologica, pp. 199-207.

Duda, A., H.-U. Schmincke 1985: Polybaric differentiation of alkali basaltic magmas: evidence from green-core clinopyroxenes (Eifel, FRG). - Contributions to Mineralogy and Petrology, 91, pp. 340-353.

Embey-Isztin, A., H.G. Scharbert, H. Dietrich, H. Poultidis 1990: Mafic granulites and clinopyroxenite xenoliths from the Transdanubian Volcanic Region (Hungary): implications for the deep structure of the Pannonian Basin. - Mineralogical Magazine, 54, pp. 463-483.

Embey-Isztin, A., H. Downes, D.E. James, B.G.J. Upton, G. Dobosi, G.A. Ingram, R.S. Harmon, H.G. Scharbert 1993: The petrogenesis of Pliocene alkaline volcanic rocks from the Pannonian Basin, Eastern Central Europe. - Journal of Petrology, 34, pp. 317-343.

Embey-Isztin, A., G. Dobosi 1995: Mantle source characteristics for Miocene-Pleistocene alkali basalts, Carpathian-Pannonian Region: A review of trace elements and isotopic composition. In: H. Downes, O. Vaselli (Eds): Neogene and Related Magmatism in the Carpatho-Pannonian Region. Acta Vulcanologica, 7/2, pp. 155-166.

Embey-Isztin, A., G. Dobosi, R. Altherr, H.-P. Meyer 2001a: Thermal evolution of the lithosphere beneath the western Pannonian Basin: evidence from deep-seated xenoliths. - Tectonophysics, 331, pp. 285-306.

Embey-Isztin, A., H. Downes, G. Dobosi 2001b: Geochemical characterization of the Pannonian Basin mantle lithosphere. - Acta Geologica Hungarica, 44, pp. 259-280.

Embey-Isztin, A., H. Downes, P.D. Kempton, G. Dobosi, M. Thirlwall 2003: Lower crustal granulite xenoliths from the Pannonian Basin, Hungary. Part 1: mineral chemistry, thermobarometry and petrology. - Contributions to Mineralogy and Petrology, 144, pp. 652-670.

Embey-Isztin, A., G. Dobosi 2007: Composition of olivines in the young alkali basalts and their peridotite xenoliths from the Pannonian Basin. - Annales Historico-Naturales Musei Nationalis Hungarici, 99, pp. 5-22.

Fodor, R.V., G. Dobosi, A.N. Sial 1995: Zoned clinopyroxenes in alkalic basalt: Clues to fractionation and magma-mixing histories for seemingly primitive magmas. - Chemie der Erde, 55, pp. 133-148.

Harangi, S., O. Vaselli, R. Kovács, S. Tonarini, N. Coradossi, D. Ferraro 1994: Volcanological and magmatological studies on the Neogene basaltic volcanoes of the Southern Little Hungarian Plain, Pannonian Basin, Western Hungary. - Mineralogica et Petrographica Acta, Bologna, 37, pp. 291-305.

Harangi, S., O. Vaselli, S. Tonarini, C. Szabó, R. Harangi, N. Coradossi 1995: Petrogenesis of Neogene extension-related alkaline volcanic rocks of the Little Hungarian Plain Volcanic Field (Western Hungary). - In: H. Downes, O. Vaselli (Eds): Neogene and Related Magmatism in the Carpatho-Pannonian Region. Acta Vulcanologica, 7/2, pp. 173-187.

Harangi, S. 2001: Neogene magmatism in the Alpine-Pannonian Transition Zone - a model for melt generation in a complex geodynamic setting. - Acta Vulcanologica, 13, pp. 25-39.

Harangi, S. 2002: New results to the knowledge of the Neogene volcanism of the Carpathian-Pannonian Region. DSc Thesis, Budapest, 290 p. (In Hungarian.)

Harangi, S., L. Lenkey 2007: Genesis of the Neogene to Quaternary volcanism in the Carpathian-Pannonian region: Role of subduction, extension, and mantle plume. - Geological Society of America Special Papers, 418, pp. 67-92.

Hirano, N., J. Yamamoto, H. Kagi, T. Ishii 2004: Young, olivine xenocryst-bearing alkali-basalt from the oceanward slope of the Japan Trench. - Contributions to Mineralogy and Petrology, 148, pp. $47-54$. 
Hoernle, K., Y.S. Zhang, D. Graham 1995: Seismic and geochemical evidence for large-scale mantle upwelling beneath the eastern Atlantic and western and central Europe. - Nature, 374, pp. 34-39.

Hofmann, A.W. 1997: Mantle geochemistry - the message from oceanic volcanism. - Nature, 385, pp. 219-229.

Jugovics, L. 1968: The Transdanubian basalt and basaltic tuff fields (in Hungarian). - MÁFI Évi Jel. 1967-ről, pp. 75-82.

Jugovics, L. 1976: The chemical character of the Hungarian basalts (in Hungarian). - MÁFI Évi Jel. 1974-ről, pp. 431-470.

Kamenetsky, V., A.J. Crawford 1998: Melt-peridotite reaction recorded in the chemistry of spinel and melt inclusions in basalt from $43^{\circ} \mathrm{N}$, Mid-Atlantic Ridge. - Earth and Planetary Science Letters, 164, pp. 345-352.

Kamenetsky, V.S., A.J. Crawford, S. Meffre 2001: Factors controlling chemistry of magmatic spinel: an empirical study of associated olivine, $\mathrm{Cr}$-spinel and melt inclusions from primitive rocks. Journal of Petrology, 42, pp. 655-671.

Kovács, I., C. Szabó 2005: Petrology and geochemistry of granulite xenoliths beneath the Nógrád-Gömör Volcanic Field, Carpathian-Pannonian Region (N-Hungary/S-Slovakia). Mineralogy and Petrology, 85, pp. 269-290.

Le Bas, M.J., R.W. Le Maitre, A. Streckeisen, B. Zanettin 1986: A chemical classification of volcanic rocks based on the total alkali-silica diagram. - Journal of Petrology, 27, pp. 745-750.

Libourel, G. 1999: Systematics of calcium partitioning between olivine and silicate melt: implications for melt structure and calcium content of magmatic olivines. - Contributions to Mineralogy and Petrology, 136, pp. 63-80.

Martin, U., K. Németh, A. Auer, C. Breitkreuz 2003: Mio-Pliocene Phreatomagmatic Volcanism in a Fluvio-Lacustrine Basin in Western Hungary. - Geolines, 15, pp. 84-90.

Morimoto, M. 1988: Nomenclature of pyroxenes. - Mineralogical Magazine, 52, pp. 535-550.

McDonough, W.F., S.S. Sun 1995: The composition of the Earth. - Chemical Geology, 120, pp. 223-253.

Natland, J.H., A.C. Adamson, C. Laverne, W.G. Melson, T. O'Hearn 1983: A compositionally nearly steady-state magma chamber at the Costa Rica Rift: evidence from basalt glass and mineral data. - Initial Reports, Deep-Sea Drilling Project, 69, pp. 811-858.

Németh, K., C. Szabó 1998: Peridotite xenolith bearing Strombolian scoria, Hawaiian spatter cones and diatremes at the Fuzes-to region in the Balaton Highland Volcanic Field, Pannonian Basin, Hungary. - IAVCEI 98, Abstract, Cape Town, RSA, pp. 43.

Németh, K., U. Martin 1999: Large hydrovolcanic field in the Pannonian Basin: general characteristics of the Bakony-Balaton Highland Volcanic Field, Hungary. - Acta Vulcanologica, 11, pp. 271-282.

Németh, K., U. Martin 1999: Late Miocene paleo-geomorphology of the Bakony-Balaton Highland Volcanic Field (Hungary) using physical volcanology data. - Zeitschrift für Geomorphologie N. F, 43, pp. 417-438.

Németh, K., U. Martin, S. Harangi 2001: Miocene phreatomagmatic volcanism at Tihany (Pannonian Basin, Hungary). - Journal of Volcanology and Geothermal Research, 111, pp. 111-135.

Ramsay, W.R.H., A.J. Crawford, J.D. Foden 1984: Field setting, mineralogy, chemistry, and genesis of arc picrites, New Georgia, Solomon Islands. - Contributions to Mineralogy and Petrology, 88, pp. 386-402.

Roeder, P.L., I. Reynolds 1991: Crystallization of chromite and chromium solubility in basaltic melts. - Journal of Petrology, 32, pp. 909-934.

Roeder, P. L. 1994: Chromite: from the fiery rain of chondrules to the Kilauea Iki lava lake. - The Canadian Mineralogist, 32/4, pp. 729-746.

Rohrbach, A., S. Schuth, C. Ballhaus, C. Münker, S. Matveev, C. Qopoto 2005: Petrological constraints on the origin of arc picrites, New Georgia Group, Solomon Islands. - Contributions to Mineralogy and Petrology, 149, pp. 685-698. 
Sági, T. 2008: Petrogenetic implications to the formation of the alkaline basalts in the western part of the Pannonian Basin based on olivine and spinel composition data (in Hungarian with English abstract). - MSc Thesis, Budapest, $93 \mathrm{pp}$

Seghedi, I., H. Downes, O. Vaselli, A. Szakács, K. Balogh, Z. Pécskay 2004: Post-collisional Tertiary-Quaternary mafic alkalic magmatism in the Carpathian-Pannonian region: a review. Tectonophysics, 393, pp. 43-62.

Shaw, C.S.J., Y. Thibault, A.D. Edgar, FE. Lloyd 1998: Mechanisms of orthopyroxene dissolution in silica-undersaturated melts at 1 atmosphere and implications for the origin of silica-rich glass in mantle xenoliths. - Contributions to Mineralogy and Petrology, 132, pp. 354-370.

Sigurdsson, H., J.-G. Schilling 1976: Spinels in Mid-Atlantic Ridge basalts: Chemistry and occurrence. - Earth and Planetary Science Letters, 29, pp. 7-20.

Simkin, T., J.V. Smith 1970: Minor-Element Distribution in Olivine. - The Journal of Geology, 78, pp. 304-325.

Smith, D. R., W. P. Leeman 2005: Chromian spinel-olivine phase chemistry and the origin of primitive basalts of the southern Washington Cascades. - Journal of Volcanology and Geothermal Research, 140, pp. 49-66.

Smith, I.E.M., S. Blake, C.J.N. Wilson, B.F. Houghton 2008: Deep-seated fractionation during the rise of a small-volume basalt magma batch: Crater Hill, Auckland, New Zealand. - Contributions to Mineralogy and Petrology, 155, pp. 511-527.

Spera, FJ. 1984: Carbon dioxide in petrogenesis III: role of volatiles in the ascent of alkaline magma with special reference to xenolith-bearing mafic lavas. - Contributions to Mineralogy and Petrology, 88, pp. 217-232.

Stormer, J.C. 1973: Calcium zoning in olivine and its relationship to silica activity and pressure. Geochimica et Cosmochimica Acta, 37, pp. 1815-1821.

Szabó, C., R.J. Bodnar 1998: Fluid-inclusion evidence for an upper-mantle origin for green clinopyroxenes in Late Cenozoic basanites from the Nógrád-Gömör Volcanic Field, Northern Hungary/Southern Slovakia. - International Geology Review, 40, pp. 765-773.

Thy, P. 1995: Experimental constraints on the evolution of transitional and mildly alkalic basalts: crystallization of spinel. - Lithos, 36, pp. 103-114.

Tracy, R.J., P. Robinson 1977: Zoned titanian augite in alkali olivine basalt from Tahiti and the nature of titanium substitutions in augite. - American Mineralogist, 62, pp. 634-645.

Wass, S.Y. 1979: Multiple origins of clinopyroxenes in alkali basaltic rocks. - Lithos, 12, pp. 115-132.

Wijbrans, J., K. Németh, U. Martin, K. Balogh 2007: ${ }^{40} \mathrm{Ar} /{ }^{39} \mathrm{Ar}$ geochronology of Neogene phreatomagmatic volcanism in the western Pannonian Basin, Hungary. - Journal of Volcanology and Geothermal Research, 164, pp. 193-204.

Woodland, A.B., P.J. Jugo 2007: A complex magmatic system beneath the Devés volcanic field, Massif Central, France: evidence from clinopyroxene megacrysts. - Contributions to Mineralogy and Petrology, 153, pp. 719-731.

Yagi, K., K. Onuma 1967: The join $\mathrm{CaMgSi}_{2} \mathrm{O}_{6}-\mathrm{CaTiAl}_{2} \mathrm{O}_{6}$ and its bearing on the titanaugites. Journal of the Faculty of Science, Hokkaido University. Series 4, Geology and mineralogy, 13, pp. $463-483$. 